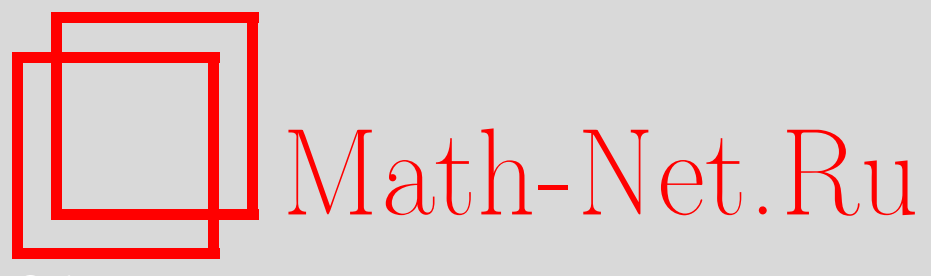

А. И. Бобенко, Ю. Б. Сурис, О принципах дискретизации дифференциальной геометрии. Геометрия сфер, УМH, 2007, том 62, выпуск 1, 3-50

DOI: https://doi.org/10.4213/rm5589

Использование Общероссийского математического портала Math-Net.Ru подразумевает, что вы прочитали и согласны с пользовательским соглашением http://www . mathnet.ru/rus/agreement

Параметры загрузки:

IP : 18.207 .199 .55

26 апреля 2023 г., 09:11:49

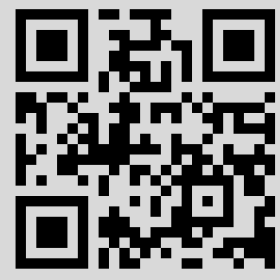




\title{
О принципах дискретизации дифференциальной геометрии. Геометрия сфер
}

\author{
А. И. Бобенко, Ю. Б. Сурис
}

Дискретная дифференциальная геометрия нацелена на создание дискретных эквивалентов понятий и методов классической дифференциальной геометрии. В данном обзоре обсуждаются следующие два фундаментальных принципа дискретизации: принцип группы преобразований (гладкие геометрические объекты и их дискретные аналоги должны быть инвариантны относительно одной и той же группы преобразований) и принцип многомерной совместности (дискретизации гладких геометрических объектов должны быть расширяемы до многомерных совместных сетей). Основная геометрическая проблема, обсуждаемая в данном обзоpe, - дискретизация поверхностей, параметризованных линиями кривизны в рамках геометрии Ли. Систематически применяя принципы дискретизации, мы находим дискретизацию непрерывной параметризации линиями кривизны, объединяющую циркулярные и конические сети.

Библиография: 62 названия.

\section{СоДЕРЖАНИЕ}

1. Введение.......................................... 4

2. Многомерная совместность как принцип дискретизации............ 13

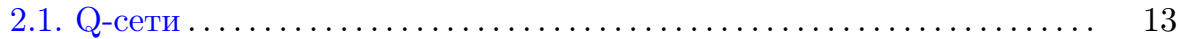

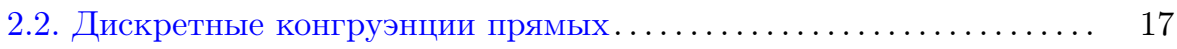

2.3. Q-сети в квадриках .............................. 20

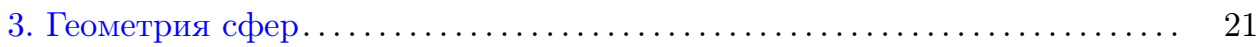

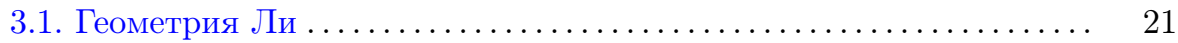

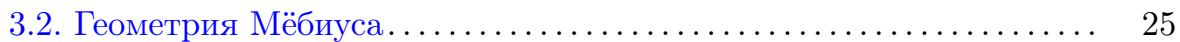

3.3. Геометрия Лагерра................................. 28

4. Дискретные линии кривизны в геометриях Ли, Мёбиуса и Лагерра.... 30

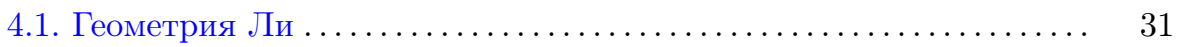

4.2. Геометрия Мёбиуса: циркулярные сети . . . . . . . . . . . . . . . 34

4.3. Геометрия Лагерра: конические сети.................. 36

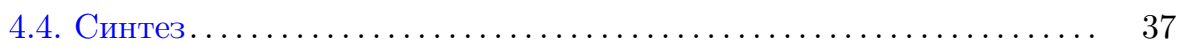

Работа выполнена при поддержке DFG Research Unit 565 "Polyhedral Surfaces" и DFG Research Center Matheon "Mathematics for key technologies", Берлин.

(С) А. И. Бовенко, Ю. Б. Сурис, 2007 


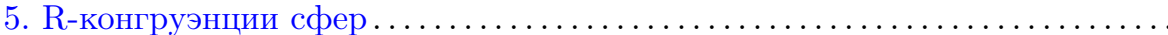

Приложение 1. Циклографическая модель геометрии Лагерра........... 46

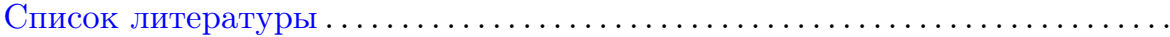

\section{1. Введение}

На стыке дифференциальной и дискретной геометрии в последние десятилетия появилась новая область - дискретная дифференциальная геометрия. В то время как в классической дифференциальной геометрии исследуются гладкие геометрические объекты, дискретная дифференциальная геометрия изучает геометрические объекты, образованные конечным количеством простейших элементов, и ставит целью разработку дискретных эквивалентов понятий и методов классической дифференциальной геометрии. Последняя, в свою очередь, вновь возникает как непрерывный предел своей дискретизации при стремлении к нулю размеров дискретных ячеек. Данная область геометрии вызывает интерес не только важными теоретическими приложениями, но и своими связями с компьютерной графикой. Важным примером этой связи служат полиэдральные поверхности, аппроксимирующие заданную гладкую поверхность.

Можно предложить много различных приемлемых дискретизаций, имеющих один и тот же непрерывный предел. Какие из них - наилучшие? С теоретической точки зрения, наилучшая дискретизация - та, которая сохраняет все фундаментальные свойства гладкой теории. Часто подобная дискретизация проясняет структуру гладкой теории и выявляет важные связи с другими областями математики (проективной геометрией, теорией интегрируемых систем, алгебраической геометрией, комплексным анализом и др.). С другой стороны, для приложений ключевым моментом является аппроксимация: наилучшая дискретизация должна обладать, по сравнению с другими дискретизациями, лучшими свойствами сходимости к гладкой модели и описывать ее дискретной моделью из небольшого числа элементов. Хотя эти теоретические и прикладные критерии наилучшей дискретизации совершенно различны, во многих случаях естественные "теоретические" дискретизации обладают также и замечательными свойствами аппроксимации и оказываются весьма полезными в приложениях [1], [2].

Указанная взаимосвязь дискретной и гладкой версий теории приводит к важным результатам как в теории поверхностей, так и в теории многогранников. Классическими достижениями дискретной дифференциальной геометрии являются фундаментальные результаты А. Д. Александрова и А. В. Погорелова в метрической теории многогранников и выпуклых поверхностей: так, теорема Александрова [3] утверждает, что любая абстрактная выпуклая полиэдральная метрика однозначно реализуется выпуклым многогранником в трехмерном евклидовом пространстве. Погорелов доказал [4] соответствующий результат о существовании и единственности реализации абстрактной выпуклой метрики с помощью аппроксимации гладкой поверхности многогранниками. 
Симплициальные поверхности, т.е. дискретные поверхности, образованные из треугольников, составляют основу компьютерной графики. Этот класс дискретных поверхностей, однако, представляется мало подходящим для аналитического исследования. Важным инструментом в теории гладких поверхностей являются (специальные) параметризации поверхности. Естественными аналогами гладких параметризованных поверхностей являются четырехугольные сети, т.е. дискретные поверхности, образованные (не обязательно плоскими) четырехугольниками. Полоски, образованные склеиванием противоположных сторон соседних четырехугольников вдоль одного из двух направлений, являются аналогами координатных линий. Вероятно, первым нетривиальным примером дискретных четырехугольных сетей, изучавшимся с этой точки зрения, являются дискретные поверхности постоянной отрицательной гауссовой кривизны, введенные Р. Зауэром и В. Вундерлихом [5], [6]. В настоящее время дискретные параметризованные поверхности приобретают все большую популярность в компьютерной графике. Они дают регулярные на вид сети, хорошо представляющие форму поверхностей [7]-[9], [2].

Как хорошо известно, дифференциальные уравнения, описывающие интересные специальные классы поверхностей и их параметризации, являются интегрируемыми (в смысле теории интегрируемых систем), и обратно, многие важные интегрируемые системы допускают дифференциально-геометрическую интерпретацию. Существенный прогресс в понимании фундаментальных структур классической дифференциальной геометрии, а одновременно и в понимании самой сути феномена интегрируемости, был обусловлен достижениями в области дискретизаций этих теорий. Как оказалось, многие сложные свойства дифференциально-геометрических объектов находят простое объяснение в рамках дискретной дифференциальной геометрии. Ранний период ее развития отражен в работах Зауэра (см. [10]). Работы А.И. Бобенко и У. Пинкаля [11], [12], А. Доливы и П. М. Сантини [13], [14] положили начало современному периоду. Тесно связанная с данной областью спектральная теория разностных операторов на графах исследовалась в работах С. П. Новикова и др. [15]-[17], см. также [18], где был развит дискретный аналог комплексного анализа на симплициальных многообразиях.

Основным примером в настоящем обзоре для нас служат дискретные поверхности в евклидовом трехмерном пространстве. Этот случай имеет все характерные черты теории во всей ее общности и допускает очевидное обобщение на многомерный случай. С другой стороны, трехмерная пространственная интуиция будет существенным подспорьем в понимании геометрических свойств теории.

Дискретная дифференциальная геометрия, тесно связанная с теорией интегрируемых систем, рассматривает в основном многомерные дискретные сети, т.е. отображения из регулярной кубической решетки $\mathbb{Z}^{m}$ в $\mathbb{R}^{N}$, обладающие определенными геометрическими свойствами (как уже упоминалось выше, мы в настоящем обзоре в основном будем интересоваться случаем $N=3$ ). В рамках этого подхода дискретные поверхности появляются как двумерные слои многомерных дискретных сетей, а их преобразования соответствуют сдвигам 
в трансверсальных направлениях решетки. Отличительной чертой теории, тем самым, является равноправие всех направлений решетки по отношению к определяющим ее геометрическим свойствам. Дискретные поверхности и их преобразования становятся неразличимы. Мы сопоставляем этому феномену свойство многомерной совместности, которая служит одним из фундаментальных принципов дискретизации. Многомерная совместность и, следовательно, существование и конструктивная реализация многомерных сетей имеют своей основой определенные теоремы инцидентности элементарной геометрии.

Концептуально можно представлять переход к непрерывному пределу как измельчение ячеек сети в некоторых из координатных направлений решетки. В этих направлениях дискретная сеть сходится к некоторым гладким поверхностям, в то время как те направления, которые остаются дискретными, соответствуют преобразованиям этих непрерывных поверхностей (см. рис. 1).
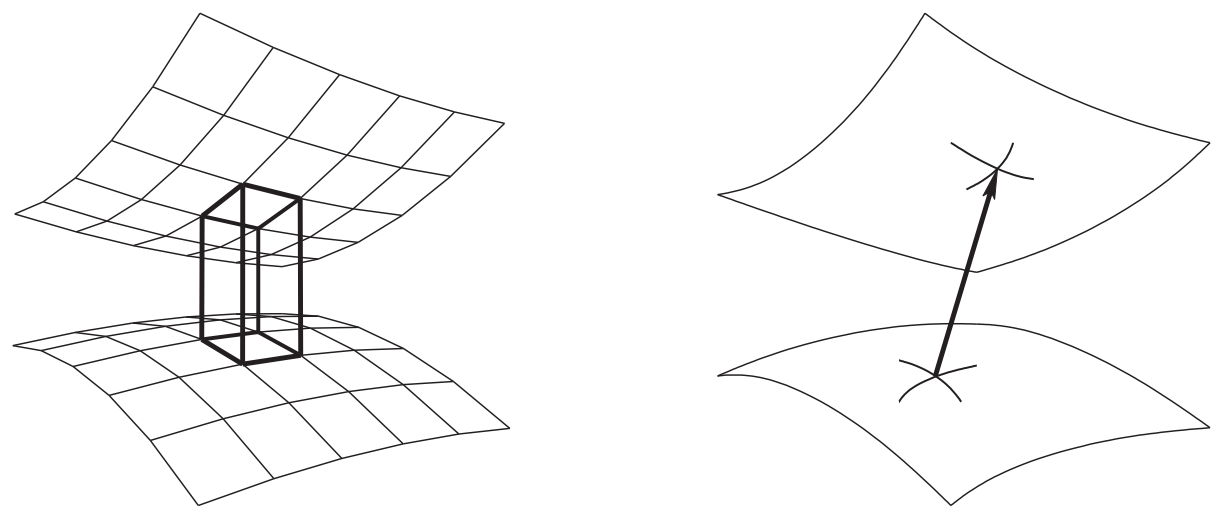

Рис. 1. От дискретной дифференциальной геометрии к классической теории поверхностей: поверхности и их преобразования появляются в результате измельчения ячеек в двух из трех координатных направлений в решетке

Гладкая теория появляется как следствие более фундаментальной дискретной теории. Подлинные корни классической теории поверхностей находятся, несколько неожиданно, среди различных теорем инцидентности элементарной геометрии. Этот феномен был выявлен для многих классов поверхностей и координатных сетей на них [19], [20], в настоящее время он получает все более широкое признание как один из фундаментальных принципов классической интегрируемой дифференциальной геометрии.

Отметим, что простое дискретное объяснение сложных дифференциальногеометрических теорий - не единственный итог исследований в этой области. Определив корни интегрируемой дифференциальной геометрии в теории многомерной совместности дискретных сетей, мы приходим к новому (геометрическому) взгляду на само понятие интегрируемости [21], [22], [20].

Простейшим и в то же время базовым примером совместной многомерной сети служат многомерные Q-сети [13], или дискретные сопряженные сети [10], выделяемые требованием плоскости всех составляющих четырехугольников. 
Указанное свойство сохраняется при проективных преобразованиях; тем самым, Q-сети являются проективными объектами (как и сопряженные сети на гладких поверхностях - их гладкие аналоги).

Здесь мы вплотную подходим к следующему базовому принципу дискретизации. В соответствии с Эрлангенской программой Ф. Клейна, геометрии классифицируются по их группам преобразований. Классическими примерами служат проективная, аффинная, евклидова, сферическая, гиперболическая геометрии, а также сферические геометрии Ли, Мёбиуса и Лагерра. Мы постулируем сохранение группы преобразований как наиболее фундаментальное свойство, которое должно соблюдаться при дискретизации. Можно рассматривать это как своего рода дискретную Эрлангенскую программу.

Тем самым мы приходим к следующим фундаментальным

\section{Принципам Дискретизации:}

1. Приниип группы преобразований: гладкие геометрические объекты и их дискретные аналоги должны принадлежать одной и той же геометрии, т.е. должны быть инвариантны относительно одной и той же группы преобразований.

2. Приниип многомерной совместности: дискретизации поверхностей, систем координат и других гладких параметризованных геометрических объектов должны быть расширяемы до многомерных совместных сетей.

Поясним, почему столь разные требования, как принцип группы преобразований и принцип совместности, могут быть одновременно наложены при дискретизации классических геометрий. Группы преобразований различных геометрий, включая геометрии Ли, Мёбиуса и Лагерра, являются подгруппами группы проективных преобразований. С классической точки зрения, подобные подгруппы описываются как группы, состоящие из проективных преобразований, сохраняющих некоторую выделенную квадрику, называемую абсолютом. Важный результат А. Доливы [23] состоит в том, что многомерные Q-сети могут быть ограничены на произвольную квадрику. Именно по этой причине принципы дискретизации работают для классических геометрий.

В настоящем обзоре мы работаем в рамках трех классических геометрий, описываемых в терминах сфер: геометрий Мёбиуса, Лагерра и Ли. Они были созданы классиками геометрии, наиболее детальное изложение можно найти в книге В. Бляшке [24].

Наиболее популярная из этих геометрий - мёбиусова. Она описывает свойства, инвариантные относительно преобразований Мёбиуса, которые порождены отражениями относительно сфер. При $N \geqslant 3$ преобразования Мёбиуса $\mathbb{R}^{N}$ совпадают с конформными преобразованиями. В геометрии Мёбиуса не различают сферы и плоскости (плоскости рассматриваются как сферы, проходящие через бесконечно удаленную точку $\infty$, что соответствует компактификации $\mathbb{R}^{N}$ до $N$-сферы $\mathbb{S}^{N}$ ). С другой стороны, точки рассматриваются как объекты, отличные от сфер. Поверхности считаются состоящими из точек. Классическими примерами понятий мёбиусовой геометрии поверхностей служат их конформные параметризации и функционал Вилмора [25]. Недавний прогресс в данной 
области в значительной степени обусловлен ее взаимосвязями с теорией интегрируемых систем [26], [27].

Геометрия Лагерра не различает точек и сфер (точки рассматриваются как сферы нулевого радиуса). С другой стороны, плоскости считаются самостоятельными элементами геометрии Лагерра. Поверхности описываются как огибающие своих касательных плоскостей. Примером преобразования Лагерра поверхности служит сдвиг всех касательных плоскостей на некоторое заданное расстояние. Это преобразование называется сдвигом вдоль нормали.

Геометрия Ли естественным образом объединяет геометрии Мёбиуса и Лагерра: точки, плоскости и сферы рассматриваются как равноправные объекты. Группа преобразований порождается преобразованиями Мёбиуса и сдвигами вдоль нормали. Поверхности описываются с помощью контактных элементов. Контактный элемент можно рассматривать как точку поверхности вместе с соответствующей касательной плоскостью. Инвариантное описание контактного элемента в геометрии Ли дает однопараметрическое семейство сфер, проходящих через данную точку и имеющих общую касательную плоскость в этой точке. Точка поверхности и касательная плоскость в этой точке - лишь два элемента этого семейства.

Некоторые аспекты теории поверхностей в геометрии Ли, связанные с интегрируемостью, изучались Е. В. Ферапонтовым [28], [29], Э. Муссо и Л. Николоди [30], а также Ф. Бурсталлом и У. Хертрих-Иеромином [31], [32].

Основная геометрическая проблема, изучаемая в настоящем обзоре, - дискретизация поверхностей, параметризованных линиями кривизны. Линии кривизны являются интегральными кривыми главных направлений. Любая поверхность вне окрестностей своих омбилических точек может быть локально параметризована линиями кривизны. Эта параметризация привлекала внимание математиков и физиков в течении двух столетий. Классические результаты в этой области отражены в монографиях Г. Дарбу [33], [34] и Л. Бьянки [35]. В частности, классический результат Ш. Дюпена [36] утверждает, что координатные поверхности триортогональной системы координат пересекаются по их общим линиям кривизны. А. Рибокур открыл преобразование поверхностей, сохраняющее линии кривизны (см. [37]). Заданная поверхность и ее преобразование Рибокура обертывают специальную сферическую конгруэнцию. Как показал Бьянки [38], преобразования Рибокура перестановочны: если даны два преобразования Рибокура одной поверхности, то существует однопараметрическое семейство их общих преобразований Рибокура (см. также [39], [40]). Е. И. Ганжа и С.П. Царев [41] установили трехмерный принцип нелинейной суперпозиции для преобразований Рибокура триортогональных систем координат.

В последние годы параметризация линиями кривизны и ортогональные системы координат вновь оказались в центре внимания математический физики как примеры интегрируемых систем. В. Е. Захаров [42] построил широкий набор явных решений с помощью метода одевания. Ортогональные системы алгебро-геометрического типа были построены И. М. Кричевером [43]. Интерес к проблеме был, в частности, вызван приложениями к теории уравнения 
ассоциативности, развитыми Б. А. Дубровиным [44]. Замечательные геометрические свойства делают параметризации поверхностей с помощью линий кривизны особенно полезными для визуализации поверхностей в компьютерной графике [7], [2].

Вопросы адекватной дискретизации параметризации поверхностей с помощью линий кривизны и дискретизация ортогональных систем координат стали в последние годы объектом интенсивного изучения. Циркулярные сети, т.е. Q-сети, в которых каждый четырехугольник-ячейка является вписанным в некоторую окружность, как дискретные аналоги поверхностей с параметризацией линиями кривизны, упоминались Э. Натборном и Р. Мартином [45]. Специальные циркулярные сети - дискретные изотермические поверхности изучались в [12]. Циркулярная дискретизация триортогональных систем координат была предложена одним из авторов в [46]. А. Долива и П. М. Сантини [13] сделали следующий важный шаг в развитии этой теории. Они рассмотрели дискретные ортогональные системы как редукцию дискретных сопряженных систем [14], обобщили их на случай произвольной размерности и доказали их многомерную совместность на основе классической теоремы Микеля [47].

Как показано в совместной работе авторов обзора и Д. Маттеса [48], циркулярные сети аппроксимируют гладкие поверхности с параметризацией линиями кривизны и ортогональные координатные системы вместе со всеми производными по параметрам любого порядка. Численные эксперименты показывают, что циркулярные сети обладают необходимыми геометрическими свойствами уже при грубом приближении, а не только в предельном приближении при измельчении размера ячеек. Это важно для приложений в компьютерной графике [2].

Удобное аналитическое описание циркулярных сетей было дано В.Г. Конопельченко и В.К. Шифом [49]. Аналитические методы теории солитонов были применены к теории циркулярных сетей А. Доливой, С. В. Манаковым

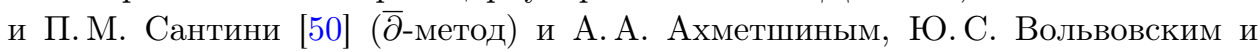
И. М. Кричевером [51] (алгебро-геометрические решения). А.И. Бобенко и У. Хертрих-Иеромин [52] дали описание циркулярных сетей в терминах алгебр Клиффорда.

Свойство циркулярности сохраняется при мебиусовых преобразованиях и тем самым должно рассматриваться как дискретизация гладкой параметризации линиями кривизны в геометрии Мёбиуса. В недавней работе Лю, Поттманна, Валлнера, Яна и Вана [2] были введены конические сети, которые следует рассматривать как дискретизацию параметризации линиями кривизны в рамках геометрии Лагерра. Это специальный класс Q-сетей, характеризуемый следующим свойством: любые четыре четырехугольника, сходящиеся в одной вершине, касаются общего конуса вращения. Конические сети можно эквивалентно характеризовать как Q-сети с циркулярным отображением Гаусса: единичные нормали к граням сети задают циркулярную сеть на единичной сфеpe $S^{2}$. Циркулярные гауссовы отображения, определенные в вершинах данной циркулярной сети, были ранее введены В.К. Шифом [53], [54], но без связи с коническими сетями. Конические сети, как и циркулярные, удовлетворяют 
второму принципу дискретизации (совместности). В настоящем обзоре путем систематического применения принципов дискретизации мы находим дискретизацию гладкой параметризации линиями кривизны, которая объединяет теорию циркулярных и конических сетей.

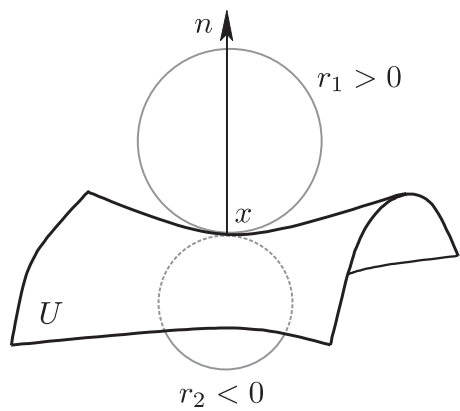

Рис. 2. Определение главных направлений с помощью соприкасающихся сфер

Хорошо известно, что линии кривизны являются объектом геометрии Ли, т.е. инвариантны относительно преобразований Мёбиуса и сдвигов вдоль нормалей. Чтобы показать это, рассмотрим инфинитезимальную окрестность $U$ точки $x$ на ориентированной гладкой поверхности в $\mathbb{R}^{3}$ и пучок сфер $S(r)$ всевозможных радиусов $r$ (с учетом ориентации, или, что то же самое, знака $r$ ), касающихся поверхности в точке $x$, см. рис. 2. Радиус $r$ считаем положительным, если $S(r)$ лежит по ту же сторону от касательной плоскости к поверхности, что и нормаль $n$, и отрицательным в противном случае; при этом $S(\infty)-$ касательная плоскость. Для малых $r_{0}>0$ сферы $S\left(r_{0}\right)$ и $S\left(-r_{0}\right)$ пересекают $U$ лишь в одной точке $x$. Множество касательных сфер с этим свойством (пересекающих $U$ лишь в $x$ ) имеет две компоненты: $M_{+}$, содержащую $S\left(r_{0}\right)$, и $M_{-}$, содержащую $S\left(-r_{0}\right)$ для малых $r_{0}>0$. Граничные значения

$$
r_{1}=\sup \left\{r: S(r) \in M_{+}\right\}, \quad r_{2}=\inf \left\{r: S(r) \in M_{-}\right\}
$$

дают главные кривизны $k_{1}=1 / r_{1}$ и $k_{2}=1 / r_{2}$ поверхности в точке $x$. Направления, по которым $S\left(r_{1}\right)$ и $S\left(r_{2}\right)$ касаются $U$, будут главными направлениями.

Как очевидно, все элементы этого описания Мёбиус-инвариантны. При сдвиге вдоль нормалей на расстояние $d$ центры сфер главных кривизн сохраняются, а их радиусы изменяются на величину сдвига $d$. Отсюда следует, что главные направления, а потому и линии кривизны, сохраняются также при сдвигах вдоль нормалей.

Ли-геометрическая природа параметризации линиями кривизны подразумевает наличие Ли-инвариантного описания. Такое описание можно найти в книге Бляшке [11]. Поверхность в геометрии Ли, как уже говорилось, считается составленной из контактных элементов. Два инфинитезимально близких контактных элемента (пучка сфер) принадлежат одной линии кривизны, если и только если они имеют общую сферу, которая и будет сферой главной кривизны. 
Дословно перенося описания Бляшке гладких поверхностей, параметризованных линиями кривизны в рамках геометрии Ли, на дискретный случай, определим главную сеть контактных элементов как отображение $\mathbb{Z}^{2} \rightarrow\{$ контактные элементы поверхностей в $\left.\mathbb{R}^{3}\right\}$ такое, что любые два соседних элемента имеют общую сферу.

В проективной модели геометрии Ли сферы в $\mathbb{R}^{3}$ (включая точки и плоскости) представляются элементами так называемой квадрики Ли $\mathbb{L} \subset \mathbb{R} \mathbb{P}^{5}$, контактные элементы представляются изотропными прямыми, т.е. прямыми в $\mathbb{L}$, поверхности представляются конгруэнциями изотропных прямых. При параметризации линиями кривизны соответствующие однопараметрические семейства прямых образуют развертывающиеся поверхности в $\mathbb{L}$.

Тем самым, дискретные главные сети контактных элементов в проективной модели геометрии Ли соответствуют дискретным конгруэнциям изотропных прямых

$$
\ell: \mathbb{Z}^{2} \rightarrow\{\text { изотропные прямые в } \mathbb{L}\}
$$

таких, что всякие две соседние прямые пересекаются. Точки пересечения соседних прямых, как и в непрерывном случае, соответствуют сферам главных кривизн. Они ставятся в соответствие ребрам $\mathbb{Z}^{2}$. Четыре сферы главных кривизн, отвечающие ребрам с общей вершиной, принадлежат одному контактному элементу, т.е. имеют общую точку касания.

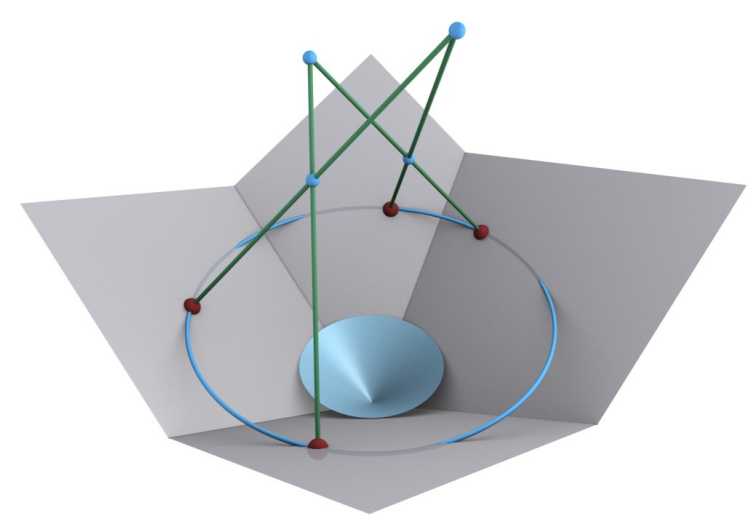

Рис. 3. Геометрия главных сетей контактных элементов. Четыре соседних элемента изображены точками и (касательными) плоскостями. Точки лежат на одной окружности, плоскости касаются конуса вращения. Соседние нормали пересекаются в центрах сфер главных кривизн

В рамках проективной геометрии дискретные конгруэнции прямых были введены А. Доливой, П. М. Сантини и М. Маньясом [55]. Дискретные конгруэнции прямых тесно связаны с Q-сетями и, подобно им, обладают многомерной совместностью. Как следует из наших результатов, они могут быть ограничены на квадрику Ли (фактически, на любую линейчатую квадрику). Таким образом, главные сети контактных элементов удовлетворяют второму 
принципу дискретизации. В частности, это обеспечивает наличие дискретных преобразований Рибокура между главными сетями контактных элементов.

Описанное выше в рамках геометрии Ли понятие главных сетей контактных элементов объединяет как соответствующее понятие в мёбиусовой геометрии (циркулярные сети), так и его аналог в геометрии Лагерра (конические сети). В самом деле, любой контактный элемент $\ell$ содержит точку $x$ и плоскость $P$. Как оказывается, для дискретной поверхности

$$
\ell: \mathbb{Z}^{2} \rightarrow\{\text { изотропные прямые в } \mathbb{L}\}=\left\{\text { контактные элементы в } \mathbb{R}^{3}\right\}
$$

точки образуют циркулярную сеть

$$
x: \mathbb{Z}^{2} \rightarrow \mathbb{R}^{3},
$$

а плоскости - коническую

$$
P: \mathbb{Z}^{2} \rightarrow\left\{\text { плоскости в } \mathbb{R}^{3}\right\} .
$$

Соответствующая конфигурация изображена на рис. 3 .

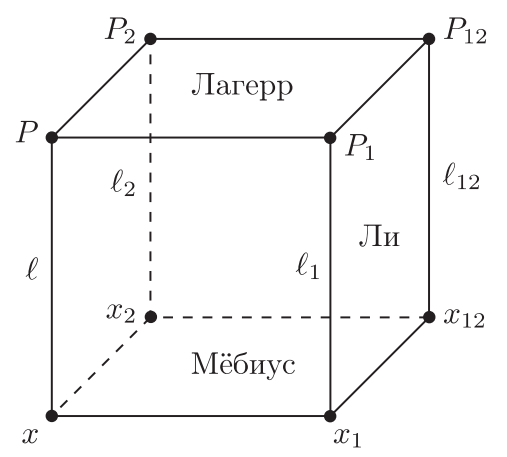

Рис. 4. Геометрия главной сети контактных элементов. Четыре соседних контактных элемента $\ell, \ell_{1}, \ell_{2}, \ell_{12}$ порождают куб с вершинами в квадрике Ли $\mathbb{L}$ и плоскими гранями. Нижний четырехугольник является пересечением трехмерного пространства $V=\operatorname{span}\left(\ell, \ell_{1}, \ell_{2}, \ell_{12}\right)$ с четырехмерным подпространством в $\mathbb{R} \mathbb{P}^{5}$, представляющим точки в $\mathbb{R}^{3}$. Верхний четырехугольник есть пересечение $V$ с четырехмерным подпространством в $\mathbb{R} \mathbb{P}^{5}$, представляющим плоскости в $\mathbb{R}^{3}$. Каждый из четырехугольников, составляющих боковую поверхность, лежит в плоскости двух пересекающихся прямых $\ell \subset \mathbb{L}$

Схематически данное соединение мёбиусовой и лагерровой геометрий в рамках геометрии Ли представлено на рис. 4.

Кратко опишем содержание обзора. В разделе 2 мы даем описание базовых многомерно совместных систем - Q-сетей и дискретных конгруэнций прямых. Основные понятия геометрий Ли, Мёбиуса и Лагерра кратко представлены в разделе 3. Раздел 4 содержит главные новые результаты о дискретных поверхностях, параметризованных линиями кривизны: определения 18, 19 в геометрии Ли и теорему 32 , описывающую взаимосвязь дискретных сетей линий 
кривизны в геометриях Ли, Мёбиуса и Лагерра. Геометрическая характеризация преобразований Рибокура и дискретных R-конгруэнций сфер как Q-сетей в квадрике Ли даны в разделе 5 .

Отметим, что ввиду наличия классического соответствия Ли между прямыми и сферами (см. [24]), теория, представленная в данном обзоре в рамках геометрии Ли, может быть перенесена в проективную геометрию прямых трехмерного пространства: квадрика Ли будет заменена квадрикой Плюккера, линии кривизны и R-конгруэнции сфер соответственно перейдут в асимптотические линии и $\mathrm{W}$-конгруэнции прямых. Проективная теория дискретных асимптотических сетей развивалась Доливой [56].

Наши исследования в дискретной дифференциальной геометрии Ли были стимулированы введением понятия конической сети в недавней работе Лю, Поттманна, Валлнера, Яна и Вана [2]. Появление второй (после циркулярных сетей) дискретизации линий кривизны поставило вопрос о связи между этими двумя дискретизациями. Независимо связь между циркулярными и коническими сетями была найдена в работе Поттманна [57]. Мы выражаем благодарность Х. Поттманну и И. Валлнеру за многочисленные сообщения по теории конических сетей и предоставление своих неопубликованных результатов. Также мы благодарим У. Пинкаля за полезные обсуждения.

\section{2. Многомерная совместность как принцип дискретизации}

2.1. Q-сети. Мы используем следующие стандартные обозначения: для функции $f$ на $\mathbb{Z}^{m}$

$$
\tau_{i} f(u)=f\left(u+e_{i}\right)
$$

где $e_{i}$ - единичный направляющий вектор $i$-й координатной оси, $1 \leqslant i \leqslant m$. Также будут использоваться краткие обозначения $f_{i}$ для $\tau_{i} f, f_{i j}$ для $\tau_{i} \tau_{j} f$ и т.д.

Наиболее общими из известных трехмерных систем, обладающих свойством 4D-совместности, являются сети, состоящие из плоских четырехугольников, или Q-сети. Двумерные Q-сети были введены Зауэром [10], многомерные обобщения предложены Доливой и Сантини [13]. Наше изложение будет следовать их работе. Фундаментальный принцип многомерной совместности для дискретных систем как основа понятия их интегрируемости был предложен в [21], [22], [20].

ОПредЕЛЕниЕ 1 (Q-сеть). Отображение $f: \mathbb{Z}^{m} \rightarrow \mathbb{R P}^{N}$ называется $m$-мерной Q-сетью (сетью плоских четырехугольников, или дискретной сопряженной сетью) в $\mathbb{R P}^{N} \quad(N \geqslant 3)$, если все ее элементарные четырехугольники $\left(f, f_{i}, f_{i j}, f_{j}\right)$ (для любого элемента $u \in \mathbb{Z}^{m}$ и любых пар $1 \leqslant i \neq j \leqslant m$ ) плоские.

Тем самым для любого четырехугольника любые четыре представителя $\widetilde{f}, \tilde{f}_{i}$, $\widetilde{f}_{j}, \widetilde{f}_{i j}$ его вершин в пространстве $\mathbb{R}^{N+1}$ однородных координат удовлетворяют уравнению вида

$$
\widetilde{f}_{i j}=c_{i j} \widetilde{f}_{j}+c_{j i} \widetilde{f}_{i}+\rho_{i j} \tilde{f}
$$


Представители вершин, лежащие в одной гиперплоскости в $\mathbb{R}^{N+1}$, например в аффинной части $\mathbb{R}^{N}$ проективного пространства $\mathbb{R} \mathbb{P}^{N}=\mathbb{P}\left(\mathbb{R}^{N+1}\right)$, удовлетворяют тому же уравнению с $1=c_{i j}+c_{j i}+\rho_{i j}$, т.е.

$$
\tilde{f}_{i j}-\tilde{f}=c_{i j}\left(\tilde{f}_{j}-\tilde{f}\right)+c_{j i}\left(\tilde{f}_{i}-\tilde{f}\right) .
$$

Если заданы три точки $f, f_{1}, f_{2}$ в $\mathbb{R} \mathbb{P}^{N}$, мы можем взять любую точку плоскости, определенной этими тремя точками как четвертую вершину $f_{12}$ элементарного четырехугольника $\left(f, f_{1}, f_{12}, f_{2}\right)$ некоторой Q-сети. Соответственно, если даны две дискретные кривые $f: \mathbb{Z} \times\{0\} \rightarrow \mathbb{R} \mathbb{P}^{N}$ и $f:\{0\} \times \mathbb{Z} \rightarrow \mathbb{R P}^{N}$ с общей точкой $f(0,0)$, можно построить бесконечно много Q-сетей $f: \mathbb{Z}^{2} \rightarrow \mathbb{R} \mathbb{P}^{N}$ с данными кривыми в качестве координатных. Построение легко осуществить по индукции, на каждом шаге мы будем иметь свободу в выборе точки на соответствующей плоскости (два вещественных параметра).

С другой стороны, построение элементарного трехмерного куба Q-сети, соответствующего элементарному трехмерному кубу решетки $\mathbb{Z}^{m}$, является корректно поставленной начальной задачей с единственным решением, поэтому естественно считать, что Q-сети описываются дискретной 3D-cuстемой (дискретной трехмерной системой):

ТЕОРема 2 (элементарный трехмерный куб Q-сети). Пусть даны семъ точек $f, f_{i}$ и $f_{i j}(1 \leqslant i<j \leqslant 3)$ в $\mathbb{R P}^{N}$ такие, что каждый из трех четьрехугольников $\left(f, f_{i}, f_{i j}, f_{j}\right)$ плоский (m.е. $f_{i j}$ лежат в плоскости $\Pi_{i j}$, определенной $\left.f, f_{i}, f_{j}\right)$. Определим три плоскости $\tau_{k} \Pi_{i j}$ тройками точек $f_{k}, f_{i k}$, $f_{j k}$ соответственно. Тогда эти три плоскости в случае общего положения пересекаются в одной точке:

$$
f_{123}=\tau_{1} \Pi_{23} \cap \tau_{2} \Pi_{13} \cap \tau_{3} \Pi_{12} .
$$

ДоказАтельство. Плоские четырехугольники $\left(f, f_{i}, f_{i j}, f_{j}\right)$ вместе со своими вершинами $f, f_{i}$ и $f_{i j}$ принадлежат трехмерному пространству $\Pi_{123}$, определенному четырьмя точками $f, f_{1}, f_{2}, f_{3}$. Тем самым плоскости $\tau_{k} \Pi_{i j}$ лежат в этом же трехмерном пространстве; в случае общего положения они имеют ровно одну точку пересечения. Теорема 2 доказана.

Элементарный шаг построения, описанный в теореме 2, схематически представлен на рис. 5. Именно этот рисунок следует представлять себе при исследовании трехмерных дискретных систем с переменными (полями) на вершинах регулярной кубической решетки.

Как вытекает из теоремы 2 , трехмерная Q-сеть $f: \mathbb{Z}^{3} \rightarrow \mathbb{R} \mathbb{P}^{N}$ полностью задается своими тремя координатными поверхностями

$$
f: \mathbb{Z}^{2} \times\{0\} \rightarrow \mathbb{R} \mathbb{P}^{N}, \quad f: \mathbb{Z} \times\{0\} \times \mathbb{Z} \rightarrow \mathbb{R P}^{N}, \quad f:\{0\} \times \mathbb{Z}^{2} \rightarrow \mathbb{R P}^{N} .
$$

Обращаясь к элементарному кубу размерности $m \geqslant 4$, легко увидеть, что мы можем задать все точки $f, f_{i}$ и $f_{i j}$ для всех $1 \leqslant i<j \leqslant m$. Действительно, эти данные явно независимы и мы можем построить все прочие вершины элементарного куба, исходя из них, при условии, что в прочессе построения не 


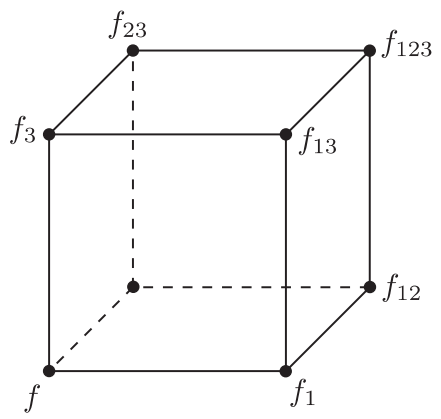

Рис. 5. 3D-система на элементарном кубе: поле в белой вершине определено полями в семи черных вершинах (начальными данными)

будет встречаться противоречия. Для демонстрации априорной возможности подобных противоречий рассмотрим подробно случай $m=4$. Исходя из $f$, $f_{i}$ и $f_{i j}(1 \leqslant i<j \leqslant 4)$, однозначно определим все $f_{i j k}$. После этого в принципе имеется четыре различных способа определить $f_{1234}$ из четырех трехмерных кубов, прилегающих к этой точке; см. рис. 6. Отсутствие противоречий и означает автоматическое совпадение этих четырех возможностей для $f_{1234}$. Мы будем называть это свойство 4D-совместностью.

ОПРЕДЕЛЕНИЕ 3 (4D-совместность). 3D-система называется 4D-совместной, если можно потребовать ее выполнения на всех трехмерных гранях элементарного куба $\mathbb{Z}^{4}$ (см. рис. 6 ).

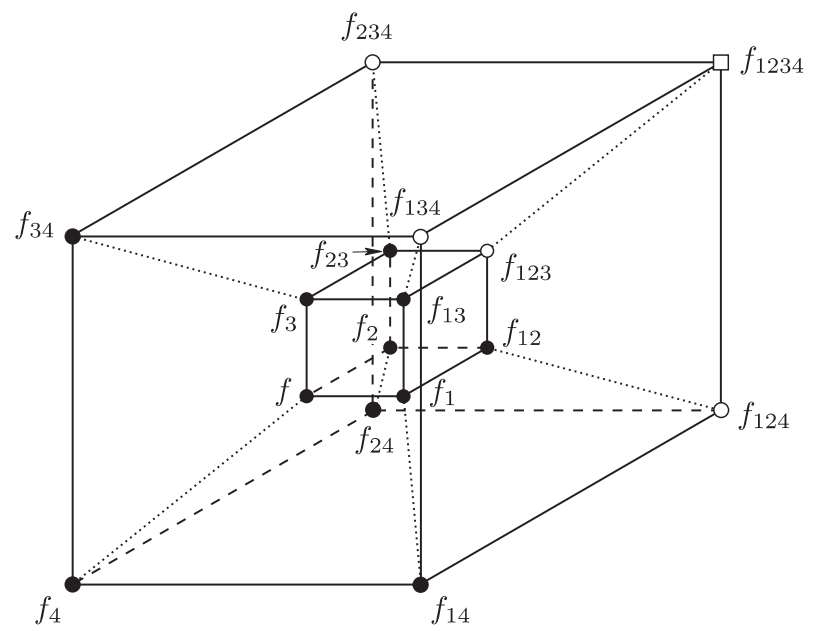

Рис. 6. 4D-совместность 3D-систем: поля в черных вершинах (начальные данные) определяют, в силу $3 \mathrm{D}$-системы, поля $f_{i j k}$ в белых кружках. Последующее применение 3D-системы дает априори четыре разных ответа для $f_{1234}$. Система является $4 \mathrm{D}$-совместной, если все эти ответы совпадают для любых начальных данных 
Замечательный факт состоит в том, что Q-сети обладают этим свойством.

Теорема 4 (Q-сети 4D-совместны). Трехмерная дискретная система, описъьвающая Q-сети, 4D-совместна.

ДокАЗАТЕЛЬСтво. В описанном выше построении одно из возможных значений $f_{1234}$ определяется как

$$
f_{1234}=\tau_{1} \tau_{2} \Pi_{34} \cap \tau_{1} \tau_{3} \Pi_{24} \cap \tau_{1} \tau_{4} \Pi_{23},
$$

три другие получаются при циклической перестановке индексов. Таким образом, нам необходимо доказать, что шесть плоскостей $\tau_{i} \tau_{j} \Pi_{k \ell}$ пересекаются в одной точке.

Прежде всего, предположим, что рассматриваемое пространство $\mathbb{R P}^{N}$ имеет размерность $N \geqslant 4$. Тогда в общем положении пространство $\Pi_{1234}$, определенное пятью точками $f, f_{i}(1 \leqslant i \leqslant 4)$, будет четырехмерным. Как легко видеть, плоскость $\tau_{i} \tau_{j} \Pi_{k \ell}$ является пересечением двух трехмерных пространств $\tau_{i} \Pi_{j k \ell}$ и $\tau_{j} \Pi_{i k \ell}$. Действительно, подпространство $\tau_{i} \Pi_{j k \ell}$, определенное четырьмя точками $f_{i}, f_{i j}, f_{i k}, f_{i \ell}$, содержит также $f_{i j k}, f_{i j \ell}$ и $f_{i k \ell}$. Поэтому как $\tau_{i} \Pi_{j k \ell}$, так и $\tau_{j} \Pi_{i k \ell}$ содержат три точки $f_{i j}, f_{i j k}$ и $f_{i j \ell}$, которые определяют плоскость $\tau_{i} \tau_{j} \Pi_{k \ell}$. Таким образом, пересечение шести плоскостей $\tau_{i} \tau_{j} \Pi_{k \ell}$ можно альтернативно описать как пересечение четырех трехмерных подпространств $\tau_{1} \Pi_{234}$, $\tau_{2} \Pi_{134}, \tau_{3} \Pi_{124}$ и $\tau_{4} \Pi_{123}$ одного и того же четырехмерного пространства $\Pi_{1234}$. Это пересечение в общем случае состоит ровно из одной точки.

В случае $N=3$ вложим нашу конфигурацию в $\mathbb{R} \mathbb{P}^{4}$, затем слегка пошевелим точку $f_{4}$, добавив небольшую величину к ее четвертой координате. Применим теперь вышеприведенные аргументы, а затем устремим четвертую координату к нулю. Как легко видеть, описанное возмущение является регулярным, что влечет утверждение о 4D-совместности и в этом случае. Теорема 4 доказана.

$m$-мерная совместность трехмерных дискретных систем определяется при $m>4$ аналогично случаю $m=4$. По достаточно общей причине, 4-мерная совместность уже влечет $m$-мерную совместность для всех $m>4$.

Tеорема 5 (4D-совместность влечет совместность во всех высших размерностях). Всякая 4D-совместная трехмерная дискретная система также будет $m$-мерно совместна для любого $m>4$.

ДокАЗАТЕЛЬСтво проведем по индукции. Для упрощения обозначений мы проведем шаг индукции лишь для $m=5$, общий случай полностью аналогичен.

Начальные данные для $3 \mathrm{D}$-системы на пятимерном кубе $\mathscr{C}_{12345}$ с полями в вершинах состоят из полей $f, f_{i}$ и $f_{i j}$ для всех $1 \leqslant i<j \leqslant 5$. Исходя из этих данных, вначале получаем десять полей $f_{i j k}$ для $1 \leqslant i<j<k \leqslant 5$, а затем пять полей $f_{i j k \ell}$ для $1 \leqslant i<j<k<\ell \leqslant 5$ (тот факт, что эти поля корректно определены, есть не что иное, как 4D-совместность на четырехмерных кубах $\left.\mathscr{C}_{i j k l}\right)$. Теперь имеется десять различных возможных значений для $f_{12345}$, происходящих из десяти $3 \mathrm{D}-$ кубов $\tau_{i} \tau_{j} \mathscr{C}_{k \ell m}$. Для доказательства совпадения этих десяти значений рассмотрим пять $4 \mathrm{D}$-кубов $\tau_{i} \mathscr{C}_{j k \ell m}$. Например, для 4D-куба 
$\tau_{1} \mathscr{C}_{2345}$ предположение теоремы - 4D-совместность - обеспечивает совпадение четырех значений $f_{12345}$, которые находятся из четырех $3 \mathrm{D}$-кубов

$$
\tau_{1} \tau_{2} \mathscr{C}_{345}, \quad \tau_{1} \tau_{3} \mathscr{C}_{245}, \quad \tau_{1} \tau_{4} \mathscr{C}_{235}, \quad \tau_{1} \tau_{5} \mathscr{C}_{234}
$$

Аналогично, для 4D-куба $\tau_{2} \mathscr{C}_{1345} 4 \mathrm{D}$-совместность приводит к выводу о совпадении четырех значений $f_{12345}$, которые можно найти из

$$
\tau_{1} \tau_{2} \mathscr{C}_{345}, \quad \tau_{2} \tau_{3} \mathscr{C}_{145}, \quad \tau_{2} \tau_{4} \mathscr{C}_{135}, \quad \tau_{2} \tau_{5} \mathscr{C}_{134}
$$

Заметим, что $3 \mathrm{D}$-куб $\tau_{1} \tau_{2} \mathscr{C}_{345}$ - пересечение $\tau_{1} \mathscr{C}_{2345}$ и $\tau_{2} \mathscr{C}_{1345}$ - присутствует в обоих списках, поэтому мы имеем семь совпадающих значений $f_{12345}$. Проводя подобные рассуждения для оставшихся 4D-кубов $\tau_{i} \mathscr{C}_{j k \ell m}$, приходим к требуемому результату. Теорема 5 доказана.

Как следует из теорем 4 и 5 , Q-сети $m$-мерно совместны при любом $m \geqslant 4$. Этот факт, в свою очередь, позволяет показать существование преобразований Q-сетей с замечательными свойствами перестановочности. Мы дадим только определение, отсылая читателя к работам [55], [20], где можно найти все необходимые детали.

ОПРЕДЕЛЕниЕ 6 (F-преобразование Q-сетей). Две $m$-мерные Q-сети $f, f^{+}$: $\mathbb{Z}^{m} \rightarrow \mathbb{R P}^{N}$ называются связанными $\mathrm{F}$-преобразованием (фундаментальным преобразованием), если все четырехугольники $\left(f, f_{i}, f_{i}^{+}, f^{+}\right)$(для любого элемента $u \in \mathbb{Z}^{m}$ и любых $\left.1 \leqslant i \leqslant m\right)$ плоские, т.е. если сеть $F: \mathbb{Z}^{m} \times\{0,1\} \rightarrow \mathbb{R P}^{N}$, формально определенная условиями $F(u, 0)=f(u)$ и $F(u, 1)=f^{+}(u)$, составляет два слоя $(m+1)$-мерной Q-сети. Также будем говорить, что эти две Q-сети являются F-преобразованием друг друга.

Из теоремы 2 следует, что если дана Q-сеть $f$, то ее F-преобразование $f^{+}$ однозначно определено, как только заданы подходящим образом выбранные точки вдоль ее координатных осей.

2.2. Дискретные конгруэнции прямых. Другим важным геометрическим объектом, описываемым 4D-совместной трехмерной системой, являются дискретные конгруэниии прямых. Теория таких конгруэнций была развита А. Доливой, П. М. Сантини и М. Маньясом [55], в настоящем разделе мы будем следовать их изложению.

Обозначим через $\mathscr{L}^{N}$ пространство прямых в $\mathbb{R} \mathbb{P}^{N}$; это пространство можно отождествить с грассманианом $\operatorname{Gr}(N+1,2)$ двумерных векторных подпространств $\mathbb{R}^{N+1}$.

ОПРЕДЕЛЕНИЕ 7 (дискретная конгруэнция прямых). Отображение $\ell: \mathbb{Z}^{m} \rightarrow$ $\mathscr{L}^{N}$ называется $m$-мерной дискретной конгруэнцией прямых в $\mathbb{R P}^{N}(N \geqslant 3)$, если любые две соседние прямые $\ell, \ell_{i}$ (для любого элемента $u \in \mathbb{Z}^{m}$ и любого $1 \leqslant i \leqslant m)$ пересекаются (компланарны).

Например, прямые $\ell=\left(f f^{+}\right)$, соединяющие соответствующие точки двух Q-сетей $f, f^{+}: \mathbb{Z}^{m} \rightarrow \mathbb{R P}^{N}$, связанных F-преобразованием, очевидным образом образуют дискретную конгруэнцию прямых. 
Дискретная конгруэнция прямых называется общей, если для любого $u \in \mathbb{Z}^{m}$ и любых $1 \leqslant i \neq j \neq k \neq i \leqslant m$ четыре прямые $\ell, \ell_{i}, \ell_{j}$ и $\ell_{k}$ порождают четырехмерное пространство (т.е. пространство максимально возможной размерности). Отсюда, в частности, получается, что для любого $u \in \mathbb{Z}^{m}$ и любых $1 \leqslant i \neq j \leqslant m$ три прямые $\ell, \ell_{i}$ и $\ell_{j}$ порождают трехмерное подпространство.

Построение конгруэнций прямых аналогично построению Q-сетей. Если даны три прямые $\ell, \ell_{1}, \ell_{2}$ конгруэнции, то имеется двухпараметрическое семейство прямых, которые могут быть взяты в качестве четвертой прямой $\ell_{12}$ : достаточно соединить прямой любую точку $\ell_{1}$ с любой точкой $\ell_{2}$. Тем самым, если даны две последовательности $\ell: \mathbb{Z} \times\{0\} \rightarrow \mathscr{L}^{N}$ и $\ell:\{0\} \times \mathbb{Z} \rightarrow \mathscr{L}^{N}$ прямых с общей прямой $\ell(0,0)$ такие, что любые две соседние прямые компланарны, мы можем расширить их до двумерной конгруэнции прямых $f: \mathbb{Z}^{2} \rightarrow \mathscr{L}^{N}$ бесконечным числом способов: на каждом шаге индуктивного построения имеется свобода в выборе прямой из двухпараметрического семейства.

Следующая теорема показывает, что невырожденные конгруэнции прямых описываются дискретной трехмерной системой.

ТЕОРема 8 (элементарный куб дискретной конгруэнции прямых). Пусть заданы семь прямых $\ell, \ell_{i}$ и $\ell_{i j}(1 \leqslant i<j \leqslant 3)$ в $\mathbb{R P}^{N}$ таких, что $\ell$ пересекает каждую из $\ell_{i}$, пространство $V_{123}$, порожденное $\ell, \ell_{1}, \ell_{2}, \ell_{3}$, четырехмерно и каждая $\ell_{i}$ пересекает обе прямые $\ell_{i j} u \ell_{i k}$. Тогда в случае общего положения существует единственная прямая $\ell_{123}$, пересекающая все три $\ell_{i j}$.

ДокАЗАТЕЛЬСтво. Все семь заданных прямых, а тем самым и трехмерные пространства $\tau_{i} V_{j k}=\operatorname{span}\left(\ell_{i}, \ell_{i j}, \ell_{i k}\right)$, лежат в $V_{123}$. Любая прямая, пересекающая все три $\ell_{i j}$, должна лежать в пересечении этих трехмерных пространств. Но в общем положении три трехмерных подпространства в $V_{123}$ пересекаются по прямой:

$$
\ell_{123}=\tau_{1} V_{23} \cap \tau_{2} V_{13} \cap \tau_{3} V_{12} .
$$

Теперь нетрудно показать, что эта прямая действительно пересекает все три $\ell_{i j}$. Например, $\tau_{1} V_{23} \cap \tau_{2} V_{13}=\operatorname{span}\left(\ell_{12}, \ell_{13}\right) \cap \operatorname{span}\left(\ell_{12}, \ell_{23}\right)$ есть плоскость, содержащая $\ell_{12}$, поэтому ее пересечение с $\tau_{3} V_{12}$ (т.е. прямая $\ell_{123}$ ) пересекает $\ell_{12}$. Теорема 8 доказана.

Сходное рассуждение доказывает следующую теорему.

TЕОРема 9 (дискретные конгруэнции прямых 4D-совместны). 3D-система, описывающая дискретные конгруэнции прямых, 4D-совместна.

Как и в случае Q-сетей, из данной теоремы вытекает существование преобразований дискретных конгруэнций прямых с замечательными свойствами перестановочности.

ОПреДЕЛЕНИЕ 10 (F-преобразование конгруэнций прямых). Две $m$-мерных конгруэнции прямых $\ell, \ell^{+}: \mathbb{Z}^{m} \rightarrow \mathscr{L}^{N}$ называются F-преобразованием друг друга, если соответствующие прямые $\ell$ и $\ell^{+}$пересекаются (для любого $u \in \mathbb{Z}^{m}$ ), т.е. если отображение $L: \mathbb{Z}^{m} \times\{0,1\} \rightarrow \mathscr{L}^{N}$, определенное формулами $L(u, 0)=$ $\ell(u)$ и $L(u, 1)=\ell^{+}(u)$, задает два слоя $(m+1)$-мерной конгруэнции прямых. 
Из теоремы 8 следует, что если дана конгруэнция прямых $\ell$, то ее F-преобразование $\ell^{+}$однозначно определено, как только подходящим образом заданы ее прямые на координатных осях соответствующей дискретной решетки.

В соответствии с определением 7 , любые две соседние прямые линии $\ell=\ell(u)$ и $\ell_{i}=\ell\left(u+e_{i}\right)$ конгруэнции пересекаются ровно в одной точке $f=\ell \cap \ell_{i} \in \mathbb{R P}^{N}$, которая тем самым комбинаторно соответствует ребру $\left(u, u+e_{i}\right)$ решетки $\mathbb{Z}^{m}$ : $f=f\left(u, u+e_{i}\right)$. Однако иногда удобнее использовать обозначение $f\left(u, u+e_{i}\right)=$ $f^{(i)}(u)$ для этой точки, связывая ее тем самым с вершиной $u$ этой решетки (и, разумеется, с координатным направлением $i$ ). См. рис. 7.

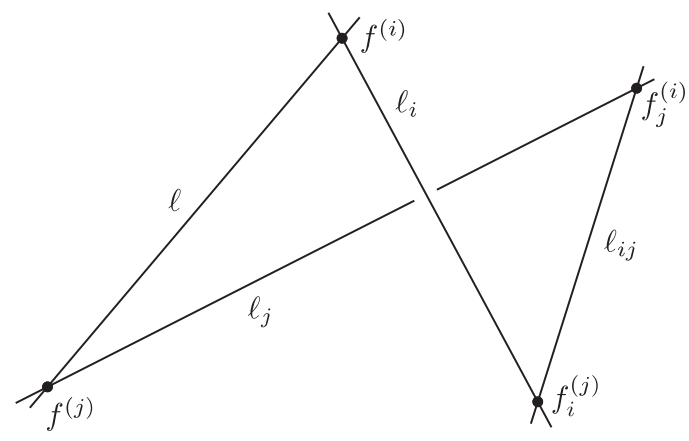

Рис. 7. Четыре прямые дискретной конгруэнции прямых

ОПРЕДЕЛЕНИЕ 11 (фокальная сеть). Для любой дискретной конгруэнции прямых $\quad \ell: \mathbb{Z}^{m} \rightarrow \mathscr{L}^{N}$ отображение $f^{(i)}: \mathbb{Z}^{m} \rightarrow \mathbb{R P}^{N}$, определенное как $f^{(i)}(u)=\ell(u) \cap \ell\left(u+e_{i}\right)$, называется ее $i$-й фокальной сетью.

ТеОрема 12. Для общей дискретной конгруэнции прямъх $\ell: \mathbb{Z}^{m} \rightarrow \mathscr{L}^{N}$ все ее фокальные сети $f^{(k)}: \mathbb{Z}^{m} \rightarrow \mathbb{R P}^{N}, 1 \leqslant k \leqslant m$, являются $Q$-сетями.

ДокАЗАТЕЛЬСТво будем проводить в два этапа.

1. Во-первых, покажем, что для $k$-й фокальной сети $f^{(k)}$ все ее элементарные четырехугольники $\left(f^{(k)}, f_{i}^{(k)}, f_{i k}^{(k)}, f_{k}^{(k)}\right)$ плоские. Это верно для любой дискретной конгруэнции прямых. Действительно, обе точки $f^{(k)}$ и $f_{k}^{(k)}$ лежат на прямой $\ell_{k}$, а обе точки $f_{i}^{(k)}$ и $f_{i k}^{(k)}-$ на прямой $\ell_{i k}$. Следовательно, все эти четыре точки лежат в плоскости, порожденной этими двумя прямыми $\ell_{k}$ и $\ell_{i k}$, которые пересекаются по определению дискретной конгруэнции прямых.

2. Во-вторых, покажем, что для $k$-й фокальной сети $f^{(k)}$ все ее элементарные четырехугольники $\left(f^{(k)}, f_{i}^{(k)}, f_{i j}^{(k)}, f_{j}^{(k)}\right)$, с $i$ не равными $j$ и отличными от $k$, плоские. Здесь мы существенно используем предположение об общности положения конгруэнции $\ell$. Все четыре рассматриваемые точки лежат в каждом из трехмерных пространств

$$
V_{i j}=\operatorname{span}\left(\ell, \ell_{i}, \ell_{j}, \ell_{i j}\right) \quad \text { и } \quad \tau_{k} V_{i j}=\operatorname{span}\left(\ell_{k}, \ell_{i k}, \ell_{j k}, \ell_{i j k}\right)
$$

(см. рис. 8). Оба эти пространства лежат в четырехмерном пространстве $V_{i j k}=$ $\operatorname{span}\left(\ell, \ell_{i}, \ell_{j}, \ell_{k}\right)$ и в общем случае пересекаются по плоскости. Теорема 12 доказана. 


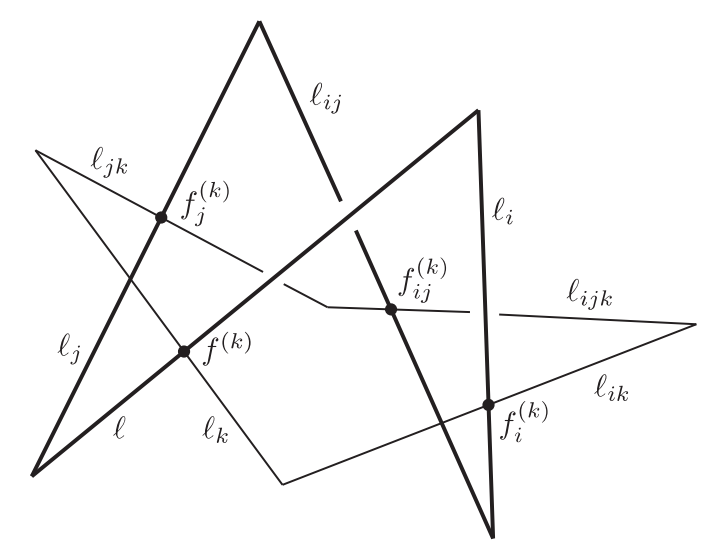

Рис. 8. Элементарный $(i j)$-четырехугольник $k$-й фокальной сети

СлЕдСтвиЕ 13 (фокальная сеть F-преобразования конгруэнции прямых). Пусть даны две общие конгруэниии прямых $\ell, \ell^{+}: \mathbb{Z}^{m} \rightarrow \mathscr{L}^{N}$, связанные между собой F-преобразованием. Тогда точки пересечения $f=\ell \cap \ell^{+}$образуют Q-cems $f: \mathbb{Z}^{m} \rightarrow \mathbb{R P}^{N}$.

2.3. Q-сети в квадриках. Здесь мы рассмотрим один важный класс допустимых редукций Q-сетей: именно, Q-сеть может быть ограничена на произвольную квадрику в $\mathbb{R P}^{N}$. В гладкой дифференциальной геометрии, т.е. для сопряженных сетей на поверхностях, аналогичный результат принадлежит Г. Дарбу [33]. В дискретной дифференциальной геометрии это было показано А. Доливой [23].

Основанием данного результата служит следующий фундаментальный факт, хорошо известный в классической проективной геометрии (см., например, [58]):

ТЕорема 14 (ассоциированная точка). Для любых семи точек общего положения в $\mathbb{C P}^{3}$ существует восъмая точка (называемая ассоциированной с даннъми), принадлежащая любой квадрике, проходящей через данные семь точек.

ДокАЗАТЕЛЬство основывается на следующем вычислении. Уравнение $\mathscr{Q}=0$ квадрики в $\mathbb{C P}^{3}$ имеет десять коэффициентов (однородный полином от четырех переменных). Следовательно, существует единственная квадрика $\mathscr{Q}=0$, проходящая через девять точек общего положения. Аналогично, существует пучок (однопараметрическое линейное семейство) квадрик $\mathscr{Q}+\lambda \mathscr{Q}^{\prime}=0$, проходящих через восемь точек общего положения, и двухпараметрическое линейное семейство квадрик $\mathscr{Q}+\lambda \mathscr{Q}^{\prime}+\mu \mathscr{Q}^{\prime \prime}=0$, проходящих через семь точек общего положения. Вновь для случая общего положения решение системы трех квадратных уравнений

$$
\mathscr{Q}=0, \quad \mathscr{Q}^{\prime}=0, \quad \mathscr{Q}^{\prime \prime}=0
$$


на координаты точек пересечения трех квадрик в $\mathbb{C P}^{3}$ состоит из восьми точек. Как можно показать, эти три квадрики, порождающие указанное выше двухпараметрическое семейство, действительно обладают требуемой общностью. Ясно, что получающиеся восемь точек лежат на каждой квадрике этого двухпараметрического семейства. Теорема 14 доказана.

Теорема 15 (элементарный куб Q-сети в квадрике). Если семь точек $f, f_{i}$ u $f_{i j}(1 \leqslant i<j \leqslant 3)$ элементарного куба $Q$-сети $f: \mathbb{Z}^{m} \rightarrow \mathbb{R P}^{N}$ лежат на квадрике $\mathscr{Q} \subset \mathbb{R P}^{N}$, то на ней же лежит и восъмая точка $f_{123}$.

ДоказАтельство. Исходные семь точек можно считать лежащими в трехмерном подпространстве, и они, очевидно, принадлежат трем (вырожденным) квадрикам - парам плоскостей $\Pi_{j k} \cup \tau_{i} \Pi_{j k}$ при $(j k)=(12),(23),(31)$. Далее, восьмая точка пересечения этих квадрик есть $f_{123}=\tau_{1} \Pi_{23} \cap \tau_{2} \Pi_{31} \cap \tau_{3} \Pi_{12}$, именно она и должна быть их ассоциированной точкой. В соответствии с теоремой 14, она принадлежит любой квадрике, содержащей заданные семь точек, в частности $\mathscr{Q}$. Теорема 15 доказана.

\section{3. Геометрия сфер}

3.1. Геометрия Ли. Классическим источником по геометрии Ли служит книга В. Бляшке [24], см. также современное изложение в книге Т. Сесиля [59].

Следующие геометрические объекты в евклидовом пространстве $\mathbb{R}^{N}$ считаются базовыми в геометрии Ли.

- Ориентированнъе гиперсферь. Гиперсфера в $\mathbb{R}^{N}$ с центром $c \in \mathbb{R}^{N}$ и радиусом $r>0$ задается уравнением $S=\left\{x \in \mathbb{R}^{N}:|x-c|^{2}=r^{2}\right\}$. Она делит $\mathbb{R}^{N}$ на две части, внутреннюю и внешнюю. Если считать одну из этих частей $\mathbb{R}^{N}$ "положительной", получаем понятие ориентированной гиперсферы. Тем самым имеются две ориентированные гиперсферы $S^{ \pm}$ для любой $S$. Можно учесть ориентацию сферы, приписав знак ее радиусу $\pm r$. Например, можно приписать положительный радиус $r>0$ гиперсферам с единичным нормальным вектором, направленным внутрь, и отрицательный $r<0$ гиперсферам с единичным нормальным вектором, направленным наружу.

- Ориентированные гиперплоскости. Гиперплоскость в $\mathbb{R}^{N}$ задается уравнением $P=\left\{x \in \mathbb{R}^{N}:\langle v, x\rangle=d\right\}$, или единичной нормалью $v \in \mathbb{S}^{N-1}$ и точкой $d \in \mathbb{R}$. Очевидно, пары $(v, d)$ и $(-v,-d)$ представляют одну и ту же гиперплоскость. Она делит $\mathbb{R}^{N}$ на два подпространства. Считая одно из этих пространств положительным, приходим к понятию ориентированной гиперплоскости. Таким образом, имеем две ориентированные гиперплоскости $P^{ \pm}$для каждой $P$. Можно зафиксировать ориентацию гиперплоскости, ассоциировав с ней пару $(v, d)$ с единичной нормалью $v$, направленной в положительное полупространство.

- Точки. Точки $x \in \mathbb{R}^{N}$ рассматриваются как гиперсферы нулевого радиyca. 
- Бесконечность. Пространство $\mathbb{R}^{N}$ компактифицируется путем добавления точки на бесконечности $\infty$ и задания базиса открытых окрестностей $\infty$, например внешности гиперсфер $|x|^{2}=r^{2}$. Определенная таким образом компактификация топологически эквивалентна сфере $\mathbb{S}^{N}$.

- Контактные элементы. Контактный элемент гиперповерхности есть пара, состоящая из точки $x \in \mathbb{R}^{N}$ и (ориентированной) гиперплоскости $P$, проходящей через $x$; вместо гиперплоскости $P$ можно использовать нормальный вектор $v$ к $P$ в $x$. В рамках геометрии Ли контактный элемент отождествляется с множеством (пучком) всех гиперсфер $S$, проходящих через $x$ и касающихся $P$ (и друг друга) с учетом ориентации, т.е. имеющих общий нормальный вектор $v$ в $x$, см. рис. 9.

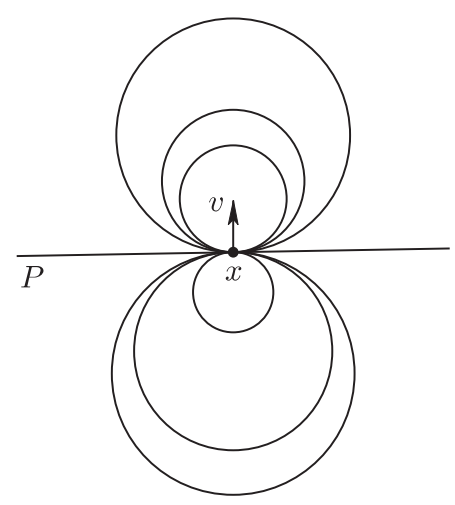

Рис. 9. Контактный элемент

Все эти объекты моделируются в геометрии Ли как точки, соответственно прямые, в $(N+2)$-мерном проективном пространстве $\mathbb{P}\left(\mathbb{R}^{N+1,2}\right)$ с сопутствующим пространством однородных координат $\mathbb{R}^{N+1,2}$. Последнее пространство порождено $N+3$ линейно независимыми векторами $\mathbf{e}_{1}, \ldots, \mathbf{e}_{N+3}$ и снабжено псевдоевклидовым скалярным произведением

$$
\left\langle\mathbf{e}_{i}, \mathbf{e}_{j}\right\rangle= \begin{cases}1, & i=j \in\{1, \ldots, N+1\} \\ -1, & i=j \in\{N+2, N+3\} \\ 0, & i \neq j .\end{cases}
$$

Удобно ввести два изотропных вектора

$$
\mathbf{e}_{0}=\frac{1}{2}\left(\mathbf{e}_{N+2}-\mathbf{e}_{N+1}\right), \quad \mathbf{e}_{\infty}=\frac{1}{2}\left(\mathbf{e}_{N+2}+\mathbf{e}_{N+1}\right),
$$

для которых

$$
\left\langle\mathbf{e}_{0}, \mathbf{e}_{0}\right\rangle=\left\langle\mathbf{e}_{\infty}, \mathbf{e}_{\infty}\right\rangle=0, \quad\left\langle\mathbf{e}_{0}, \mathbf{e}_{\infty}\right\rangle=-\frac{1}{2}
$$

Модели указанных выше объектов в пространстве $\mathbb{R}^{N+1,2}$ однородных координат таковы: 
- Ориентированная гиперсфера с иентром $c \in \mathbb{R}^{N}$ и радиусом $r \in \mathbb{R}$ (положительным или отрицательным в зависимости от ориентации):

$$
\widehat{s}=c+\mathbf{e}_{0}+\left(|c|^{2}-r^{2}\right) \mathbf{e}_{\infty}+r \mathbf{e}_{N+3} .
$$

- Ориентированная гиперплоскость $\langle v, x\rangle=d$ c $v \in \mathbb{S}^{N-1} u d \in \mathbb{R}$ :

$$
\widehat{p}=v+0 \cdot \mathbf{e}_{0}+2 d \mathbf{e}_{\infty}+\mathbf{e}_{N+3} .
$$

- Точка $x \in \mathbb{R}^{N}$ :

$$
\widehat{x}=x+\mathbf{e}_{0}+|x|^{2} \mathbf{e}_{\infty}+0 \cdot \mathbf{e}_{N+3} .
$$

- Бесконечность $\infty$ :

$$
\widehat{\infty}=\mathbf{e}_{\infty}
$$

- Контактный элемент $(x, P)$ :

$$
\operatorname{span}(\widehat{x}, \widehat{p}) .
$$

В проективном пространстве $\mathbb{P}\left(\mathbb{R}^{N+1,2}\right)$ первые четыре типа объектов представляются классами эквивалентности (4)-(7) по отношению $\xi \sim \eta \Leftrightarrow \xi=\lambda \eta$ с $\lambda \in \mathbb{R}^{*}$ и $\xi, \eta \in \mathbb{R}^{N+1,2}$. Контактный элемент представляется прямой в $\mathbb{P}\left(\mathbb{R}^{N+1,2}\right)$, проходящей через точки с представителями $\widehat{x}$ и $\widehat{p}$. Приведем некоторые важные для нас свойства этих моделей объектов.

(i) Все указанные выше объекты принадлежат квадрике $Л u \mathbb{P}\left(\mathbb{L}^{N+1,2}\right)$, где

$$
\mathbb{L}^{N+1,2}=\left\{\xi \in \mathbb{R}^{N+1,2}:\langle\xi, \xi\rangle=0\right\} .
$$

Более того, точки $\mathbb{P}\left(\mathbb{L}^{N+1,2}\right)$ находятся во взаимно однозначном соответствии с ориентированными гиперсферами в $\mathbb{R}^{N}$, включая вырожденные случаи: собственно гиперсферы соответствуют точкам $\mathbb{P}\left(\mathbb{L}^{N+1,2}\right)$ с обеими ненулевыми $\mathbf{e}_{0}$ - и $\mathbf{e}_{N+3}$-компонентами, гиперплоскости соответствуют точкам $\mathbb{P}\left(\mathbb{L}^{N+1,2}\right)$ с нулевой $\mathbf{e}_{0}$-компонентой, точки соответствуют точкам $\mathbb{P}\left(\mathbb{L}^{N+1,2}\right)$ с нулевой $\mathbf{e}_{N+3}$-компонентой, а бесконечность соответствует единственной точке $\mathbb{P}\left(\mathbb{L}^{N+1,2}\right)$, у которой обе $\mathbf{e}_{0}$ - и $\mathbf{e}_{N+3}$-компоненты нулевые.

(ii) Две ориентированные гиперсферы $S_{1}, S_{2}$ касаются с учетом ориентации (т.е. касаются друг друга и нормали в точке касания сонаправлены), если и только если

$$
\left|c_{1}-c_{2}\right|^{2}=\left(r_{1}-r_{2}\right)^{2},
$$

что эквивалентно $\left\langle\widehat{s}_{1}, \widehat{s}_{2}\right\rangle=0$.

(iii) Ориентированная гиперсфера $S=\left\{x \in \mathbb{R}^{N}:|x-c|^{2}=r^{2}\right\}$ касается ориентированной гиперплоскости $P=\left\{x \in \mathbb{R}^{N}:\langle v, x\rangle=d\right\}$ с учетом ориентации, если и только если

$$
\langle c, v\rangle-r-d=0 .
$$

В самом деле, уравнение гиперплоскости $P$, касающейся $S$ в $x_{0} \in S$, следующее: $\left\langle x_{0}-c, x-c\right\rangle=r^{2}$. Обозначив $v=\left(c-x_{0}\right) / r$ единичный 
нормальный вектор гиперплоскости $P$ (напомним, что положительные радиусы приписаны сферам с единичными нормалями, направленными внутрь), запишем это уравнение как $\langle v, x\rangle=d$ с $d=\left\langle c,\left(c-x_{0}\right) / r\right\rangle-r=$ $\langle c, v\rangle-r$, что и дает уравнение (11). Последнее уравнение эквивалентно условию $\langle\widehat{s}, \widehat{p}\rangle=0$.

(iv) Точка $x$ может рассматриваться как гиперсфера нулевого радиуса $r=0$ (в этом случае обе ориентированные гиперсферы совпадают). Отношение инцидентности $x \in S$ с гиперсферой $S$ (соответственно $x \in P$ с гиперплоскостью $P$ ) можно интерпретировать как частный случай ориентированного касания сферы радиуса $r=0$ с $S$ (соответственно с $P$ ); оно имеет место, если и только если $\langle\widehat{x}, \widehat{s}\rangle=0$ (соответственно $\langle\widehat{x}, \widehat{p}\rangle=0)$.

(v) Для гиперплоскости $P$ выполняется соотношение $\langle\widehat{\infty}, \widehat{p}\rangle=0$. Поэтому можно интерпретировать гиперплоскости как гиперсферы (бесконечного радиуса), проходящие через $\infty$. Точнее, можно интерпретировать гиперплоскость $\langle v, x\rangle=d$ как предел, при $r \rightarrow \infty$, гиперсфер радиуса $r$ с центрами на $c=r v+u \mathrm{c}\langle v, u\rangle=d$. В самом деле, для представителей (4) этих сфер имеем:

$$
\begin{aligned}
\widehat{s} & =(r v+u)+\mathbf{e}_{0}+(2 d r+\langle u, u\rangle) \mathbf{e}_{\infty}+r \mathbf{e}_{N+3} \\
& \sim(v+O(1 / r))+(1 / r) \mathbf{e}_{0}+(2 d+O(1 / r)) \mathbf{e}_{\infty}+\mathbf{e}_{N+3} \\
& =\widehat{p}+O(1 / r) .
\end{aligned}
$$

По тем же причинам бесконечность $\infty$ может рассматриваться как предельное положение любой последовательности точек $x$ с $|x| \rightarrow \infty$.

(vi) Любые две гиперсферы $S_{1}, S_{2}$, касающиеся с учетом ориентации, определяют контактный элемент (их точку касания и их общую касательную гиперплоскость). Для их представителей $\widehat{s}_{1}, \widehat{s}_{2}$ в $\mathbb{R}^{N+1,2}$ прямая в $\mathbb{P}\left(\mathbb{R}^{N+1,2}\right)$, проходящая через соответствующие точки в $\mathbb{P}\left(\mathbb{L}^{N+1,2}\right)$, изотропна, т.е. лежит целиком в квадрике Ли $\mathbb{P}\left(\mathbb{L}^{N+1,2}\right)$. Это вытекает из

$$
\left\langle\alpha_{1} \widehat{s}_{1}+\alpha_{2} \widehat{s}_{2}, \alpha_{1} \widehat{s}_{1}+\alpha_{2} \widehat{s}_{2}\right\rangle=2 \alpha_{1} \alpha_{2}\left\langle\widehat{s}_{1}, \widehat{s}_{2}\right\rangle=0 .
$$

Подобная прямая содержит единственную точку, чей представитель $\widehat{x}$ имеет нулевую $\mathbf{e}_{N+3}$-компоненту (и соответствует $x$, общей точке контакта всех гиперсфер), и, если $x \neq \infty$, единственную точку, чей представитель имеет нулевую $\mathbf{e}_{0}$-компоненту (и соответствует $P$, общей касательной гиперплоскости всех гиперсфер). В случае, когда изотропная прямая содержит $\widehat{\infty}$, все ее точки представляют параллельные гиперплоскости, которые образуют контактный элемент, проходящий через $\infty$.

Итак, если мы рассмотрим гиперплоскости как гиперсферы бесконечного радиуса, а точки - как гиперсферы нулевого радиуса, можно заключить, что:

(i) ориентированные гиперсферы находятся во взаимно однозначном соответствии с точками квадрики Ли $\mathbb{P}\left(\mathbb{L}^{N+1,2}\right)$ в проективном пространстве $\mathbb{P}\left(\mathbb{R}^{N+1,2}\right) ;$

(ii) ориентированное касание двух ориентированных гиперсфер соответствует ортогональности (любых) представителей соответствующих им точек в $\mathbb{P}\left(\mathbb{R}^{N+1,2}\right)$; 
(iii) контактные элементы гиперповерхностей находятся во взаимно однозначном соответствии с изотропными прямыми в $\mathbb{P}\left(\mathbb{R}^{N+1,2}\right)$; мы будем обозначать множество всех таких прямых через $\mathscr{L}_{0}^{N+1,2}$.

В соответствии с Эрлангенской программой Ф. Клейна, геометрия Ли изучает свойства преобразований, которые отображают ориентированные гиперсферы (включая точки и гиперплоскости) на ориентированные гиперсферы и, кроме того, сохраняют ориентированное касание пар гиперсфер. В описанной выше проективной модели, геометрия Ли изучает проективные преобразования $\mathbb{P}\left(\mathbb{R}^{N+1,2}\right)$, которые оставляют $\mathbb{P}\left(\mathbb{L}^{N+1,2}\right)$ инвариантной и, кроме того, сохраняют ортогональность точек $\mathbb{P}\left(\mathbb{L}^{N+1,2}\right)$ (что надо понимать как ортогональность их представителей в $\mathbb{L}^{N+1,2} \subset \mathbb{R}^{N+1,2}$; ясно, что это отношение ортогональности не зависит от выбора представителей). Эти преобразования называются сферическими преобразованиями Ли.

ТЕОРема 16 (основная теорема геометрии Ли).

а) Группа сферических преобразований Ли изоморфна $O(N+1,2) /\{ \pm I\}$.

b) Каждый дифбеоморфизм $\mathbb{P}\left(\mathbb{L}^{N+1,2}\right)$, сохраняющий класс изотропных прямых, есть ограничение на $\mathbb{P}\left(\mathbb{L}^{N+1,2}\right)$ сферического преобразования Ли.

Поскольку (не)обращение в нуль $\mathbf{e}_{0}$ - или $\mathbf{e}_{N+3}$-компоненты точки $\mathbb{P}\left(\mathbb{L}^{N+1,2}\right)$ не инвариантно относительно общего сферического преобразования Ли, в геометрии Ли не различают ориентированные гиперсферы, ориентированные гиперплоскости и точки.

3.2. Геометрия Мёбиуса. Книга Бляшке [24] также может служить классическим источником по геометрии Мёбиуса, современное изложение можно найти в [39].

Геометрия Мёбиуса представляет собой подгеометрию геометрии Ли, где точки выделены как сферы нулевого радиуса. Тем самым, геометрия Мёбиуса изучает свойства гиперсфер, инвариантные относительно подгруппы сферических преобразований Ли, сохраняющих множество точек. В проективной модели точки $\mathbb{R}^{N}$ выделяются как точки $\mathbb{P}\left(\mathbb{L}^{N+1,2}\right)$ с нулевой $\mathbf{e}_{N+3}$-компонентой. (Разумеется, можно заменить здесь $\mathbf{e}_{N+3}$ любым другим времениподобным вектором.) Таким образом, геометрия Мёбиуса изучает подгруппу группы сферических преобразований Ли, сохраняющих подмножество в $\mathbb{P}\left(\mathbb{L}^{N+1,2}\right)$ с нулевой $\mathbf{e}_{N+3}$-компонентой. Следующие геометрические объекты в $\mathbb{R}^{N}$ являются элементами геометрии Мёбиуса:

- (неориентированные) гиперсферы $S=\left\{x \in \mathbb{R}^{N}:|x-c|^{2}=r^{2}\right\}$ с центрами $c \in \mathbb{R}^{N}$ и радиусами $r>0$;

- (неориентированные) гиперплоскости $P=\left\{x \in \mathbb{R}^{N}:\langle v, x\rangle=d\right\}$ с единичными нормалями $v \in \mathbb{S}^{N-1}$ и $d \in \mathbb{R}$;

- точки $x \in \mathbb{R}^{N}$

- бесконечность $\infty$, компактифицирующая $\mathbb{R}^{N}$ в $\mathbb{S}^{N}$.

Строя модели этих элементов, можно использовать их описание в геометрии Ли, просто опуская $\mathbf{e}_{N+3}$-компоненту. Полученные объекты будут точками $(N+1)$-мерного пространства $\mathbb{P}\left(\mathbb{R}^{N+1,1}\right)$ с пространством однородных координат $\mathbb{R}^{N+1,1}$. Последнее пространство порождено $N+2$ линейно независимыми 
векторами $\mathbf{e}_{1}, \ldots, \mathbf{e}_{N+2}$ и снабжено скалярным произведением Минковского

$$
\left\langle\mathbf{e}_{i}, \mathbf{e}_{j}\right\rangle= \begin{cases}1, & i=j \in\{1, \ldots, N+1\} \\ -1, & i=j=N+2 \\ 0, & i \neq j .\end{cases}
$$

Мы по-прежнему будем использовать обозначения (3) в контексте геометрии Мёбиуса. Ее объекты моделируются в пространстве $\mathbb{R}^{N+1,1}$ однородных координат следующим образом.

- Гиперсфера с иентром $c \in \mathbb{R}^{N}$ и радиусом $r>0$ :

$$
\widehat{s}=c+\mathbf{e}_{0}+\left(|c|^{2}-r^{2}\right) \mathbf{e}_{\infty} .
$$

- Гиперплоскость $\langle v, x\rangle=d$ c $v \in \mathbb{S}^{N-1} u d \in \mathbb{R}:$

$$
\widehat{p}=v+0 \cdot \mathbf{e}_{0}+2 d \mathbf{e}_{\infty} .
$$

- Точка $x \in \mathbb{R}^{N}$ :

$$
\widehat{x}=x+\mathbf{e}_{0}+|x|^{2} \mathbf{e}_{\infty}
$$

- Бесконечность $\infty$ :

$$
\widehat{\infty}=\mathbf{e}_{\infty} .
$$

В проективном пространстве $\mathbb{P}\left(\mathbb{R}^{N+1,1}\right)$ эти элементы представляются точками - классами эквивалентности (12)-(15) с обычным отношением эквивалентности $\xi \sim \eta \Leftrightarrow \xi=\lambda \eta$ с $\lambda \in \mathbb{R}^{*}$ для $\xi, \eta \in \mathbb{R}^{N+1,1}$. Фундаментальные свойства приведенных выше моделей:

(i) Бесконечность $\widehat{\infty}$ можно рассматривать как предел любой последовательности $\widehat{x}$ для $x \in \mathbb{R}^{N}$ с $|x| \rightarrow \infty$. Элементы $x \in \mathbb{R}^{N} \cup\{\infty\}$ взаимно однозначно соответствуют точкам проективизированного светового конуса $\mathbb{P}\left(\mathbb{L}^{N+1,1}\right)$, где

$$
\mathbb{L}^{N+1,1}=\left\{\xi \in \mathbb{R}^{N+1,1}:\langle\xi, \xi\rangle=0\right\} .
$$

Точки $x \in \mathbb{R}^{N}$ соответствуют точкам $\mathbb{P}\left(\mathbb{L}^{N+1,1}\right)$ с ненулевой $\mathbf{e}_{0}$-компонентой, а $\infty$ соответствует единственной точке $\mathbb{P}\left(\mathbb{L}^{N+1,1}\right)$ с нулевой $\mathbf{e}_{0}$ компонентой.

(ii) Гиперсферы $\widehat{s}$ и гиперплоскости $\widehat{p}$ принадлежат $\mathbb{P}\left(\mathbb{R}_{\text {out }}^{N+1,1}\right)$, где

$$
\mathbb{R}_{\text {out }}^{N+1,1}=\left\{\xi \in \mathbb{R}^{N+1,1}:\langle\xi, \xi\rangle>0\right\}
$$

есть множество пространственно-подобных векторов пространства Минковского $\mathbb{R}^{N+1,1}$. Гиперплоскости можно представлять как гиперсферы (бесконечного радиуса), проходящие через $\infty$.

(iii) Две гиперсферы $S_{1}, S_{2}$ с центрами $c_{1}, c_{2}$ и радиусами $r_{1}, r_{2}$ ортогонально пересекаются, если и только если

$$
\left|c_{1}-c_{2}\right|^{2}=r_{1}^{2}+r_{2}^{2},
$$


что эквивалентно условию $\left\langle\widehat{s}_{1}, \widehat{s}_{2}\right\rangle=0$. Аналогично, гиперсфера $S$ ортогонально пересекается с гиперплоскостью $P$, если и только если ее центр лежит в $P$ :

$$
\langle c, v\rangle-d=0
$$

что эквивалентно условию $\langle\widehat{s}, \widehat{p}\rangle=0$.

(iv) Точку $x$ можно рассматривать как предельный случай гиперсферы нулевого радиуса $r=0$. Отношение инцидентности точки $x \in S$ и сферы $S$ (соответственно $x \in P$ и гиперплоскости $P$ ) можно интерпретировать как частный случай ортогонального пересечения сферы радиуса $r=0$ и $S$ (соответственно $P$ ), а оно имеет место, если и только если $\langle\widehat{x}, \widehat{s}\rangle=0$ (соответственно $\langle\widehat{x}, \widehat{p}\rangle=0)$.

Отметим, что гиперсферу $S$ можно также интерпретировать как множество точек $x \in S$. Соответственно, она допускает, наряду с представлением $\widehat{s}$, дуальное представление в виде трансверсального сечения $\mathbb{P}\left(\mathbb{L}^{N+1,1}\right)$ проективным $N$-мерным пространством $\mathbb{P}\left(\widehat{s}^{\perp}\right)$, полярным точке $\widehat{s}$ относительно $\mathbb{P}\left(\mathbb{L}^{N+1,1}\right)$; здесь, разумеется, $\widehat{s}^{\perp}=\left\{\widehat{x} \in \mathbb{R}^{N+1,1}:\langle\widehat{s}, \widehat{x}\rangle=0\right\}$. Это представление можно обобщить на сферы меньшей размерности.

- Cферь. $k$-мерная сфера есть пересечение $N-k$ гиперсфер $S_{i}(i=1, \ldots$, $N-k$ ) общего положения. Пересечение $N-k$ гиперсфер, представленных $\widehat{s}_{i} \in \mathbb{R}_{\text {out }}^{N+1,1}(i=1, \ldots, N-k)$, будет пересечением общего положения, если $(N-k)$-мерное линейное подпространство $\mathbb{R}^{N+1,1}$, порожденное $\widehat{s}_{i}$, пространственно-подобно:

$$
\Sigma=\operatorname{span}\left(\widehat{s}_{1}, \ldots, \widehat{s}_{N-k}\right) \subset \mathbb{R}_{\text {out }}^{N+1,1} .
$$

Как множество точек, эта $k$-сфера представляется $\mathbb{P}\left(\mathbb{L}^{N+1,1} \cap \Sigma^{\perp}\right)$, где

$$
\Sigma^{\perp}=\bigcap_{i=1}^{N-k} \widehat{s}_{i}^{\perp}=\left\{\widehat{x} \in \mathbb{R}^{N+1,1}:\left\langle\widehat{s}_{1}, \widehat{x}\right\rangle=\cdots=\left\langle\widehat{s}_{N-k}, \widehat{x}\right\rangle=0\right\}
$$

- $(k+2)$-мерное линейное подпространство $\mathbb{R}^{N+1,1}$ сигнатуры $(k+1,1)$. Через любые $k+2$ точки $x_{1}, \ldots, x_{k+2} \in \mathbb{R}^{N}$ общего положения можно провести единственную $k$-сферу. Она соответствует $(k+2)$-мерному линейному подпространству

$$
\Sigma^{\perp}=\operatorname{span}\left(\widehat{x}_{1}, \ldots, \widehat{x}_{k+2}\right)
$$

сигнатуры $(k+1,1)$ с $k+2$ линейно независимыми изотропными векторами $\widehat{x}_{1}, \ldots, \widehat{x}_{k+2} \in \mathbb{L}^{N+1,1}$. В полярном представлении, эти $k$-сферы соответствуют $(N-k)$-мерным пространственно-подобным линейным подпространствам

$$
\Sigma=\bigcap_{i=1}^{k+2} \widehat{x}_{i}^{\perp}=\left\{\widehat{s} \in \mathbb{R}^{N+1,1}:\left\langle\widehat{s}, \widehat{x}_{1}\right\rangle=\cdots=\left\langle\widehat{s}, \widehat{x}_{k+2}\right\rangle=0\right\} .
$$

Геометрия Мёбиуса изучает свойства (неориентированных) гиперсфер, инвариантные относительно проективных преобразований $\mathbb{P}\left(\mathbb{R}^{N+1,1}\right)$, которые отображают точки в точки, т.е. оставляют $\mathbb{P}\left(\mathbb{L}^{N+1,1}\right)$ инвариантным. Подобные преобразования называются преобразованиями Мёбиуса. 
ТЕоРемА 17 (фундаментальная теорема Мёбиусовой геометрии).

а) Группа преобразований Мёбиуса изоморбна $O(N+1,1) /\{ \pm I\} \simeq O^{+}(N+1,1)$, группе лоренцевых преобразований $\mathbb{R}^{N+1,1}$, сохраняющих пространственно-подобное направление.

b) Каждый конформный дифбеоморфизм $\mathbb{S}^{N} \simeq \mathbb{R}^{N} \cup\{\infty\}$ при $N \geqslant 3$ индуиирован ограничением на $\mathbb{P}\left(\mathbb{L}^{N+1,1}\right)$ преобразования Мёбиуса.

Группа $O^{+}(N+1,1)$ порождена отражениями

$$
A_{\widehat{s}}: \mathbb{R}^{N+1,1} \rightarrow \mathbb{R}^{N+1,1}, \quad A_{\widehat{s}}(\widehat{x})=\widehat{x}-\frac{2\langle\widehat{s}, \widehat{x}\rangle}{\langle\widehat{s}, \widehat{s}\rangle} \widehat{s}
$$

Если $\widehat{s}$ - гиперсфера (12), то преобразование, индуцированное $A_{\widehat{s}}$ на $\mathbb{R}^{N}$, получается из (20) простым вычислением с представителями (14) для точек и задается формулой

$$
x \mapsto c+\frac{r^{2}}{|x-c|^{2}}(x-c)
$$

(инверсия относительно сферы $S=\left\{x \in \mathbb{R}^{N}:|x-c|^{2}=r^{2}\right\}$ ); аналогично, если $\widehat{s}=\widehat{p}$ - гиперплоскость (13), то преобразование, индуцированное $A_{\widehat{p}}$ на $\mathbb{R}^{N}$, как легко вычислить, будет

$$
x \mapsto x-\frac{2(\langle v, x\rangle-d)}{\langle v, v\rangle} v
$$

(отражение относительно гиперплоскости $P=\left\{x \in \mathbb{R}^{N}:\langle v, x\rangle=d\right\}$ ).

Поскольку зануление $\mathbf{e}_{\infty}$-компоненты точки в $\mathbb{P}\left(\mathbb{R}^{N+1,1}\right)$ не инвариантно относительно общих мёбиусовых преобразований, в геометрии Мёбиуса нет различий между гиперсферами и гиперплоскостями.

3.3. Геометрия Лагерра. Вновь книга Бляшке [24] служит незаменимым классическим источником по геометрии Лагерра. Современное изложение можно найти, например, в [60], [59], [61].

Геометрия Лагерра есть подгеометрия геометрии Ли, в которой гиперплоскости выделены среди всех гиперсфер как проходящие через $\infty$. Тем самым, геометрия Лагерра изучает свойства гиперсфер, инвариантные относительно подгруппы сферических преобразований Ли, сохраняющих множество гиперплоскостей. Следующие объекты в $\mathbb{R}^{N}$ служат базовыми объектами геометрии Лагерра.

- (Ориентированные) гиперсферы $S=\left\{x \in \mathbb{R}^{N}:|x-c|^{2}=r^{2}\right\}$ с центрами $c \in \mathbb{R}^{N}$ и радиусами $r \in \mathbb{R}$ с учетом знака, они могут быть поставлены в соответствие $(N+1)$-мерным элементам $(c, r)$.

- Точки $x \in \mathbb{R}^{N}$ рассматриваются как гиперсферы радиуса нуль, они ставятся в соответствие $(N+1)$-мерным элементам $(x, 0)$.

- (Ориентированные) гиперплоскости $P=\left\{x \in \mathbb{R}^{N}:\langle v, x\rangle=d\right\}$, с единичной нормалью $v \in \mathbb{S}^{N-1}$ и $d \in \mathbb{R}$, могут быть поставлены в соответствие $(N+1)$-мерным элементам $(v, d)$.

В проективной модели геометрии Ли гиперплоскости выделяются как элементы $\mathbb{P}\left(\mathbb{L}^{N+1,2}\right)$ с нулевой $\mathbf{e}_{0}$-компонентой. (Разумеется, можно заменить

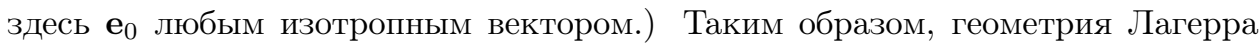


изучает подгруппу сферических преобразований Ли, сохраняющих подмножество $\mathbb{P}\left(\mathbb{L}^{N+1,2}\right)$ с нулевой $\mathbf{e}_{0}$-компонентой.

Насколько известно, не существует модели геометрии Лагерра, в которой гиперсферы и гиперплоскости могли бы быть представлены как точки одного и того же пространства. В зависимости от того, какой из этих двух типов элементов моделируется точками, приходим либо к иилиндрической модели Бляшке, либо к ииклографической модели геометрии Лагерра. Мы будем использовать первую из них, которая имеет более простое описание выделенных объектов геометрии Лагерра, т.е. гиперплоскостей. Главное преимущество второй модели - более простое описание группы преобразований Лагерра.

В обеих моделях рассматриваются два $(N+1)$-мерных проективных пространства, чьи пространства однородных координат $\mathbb{R}^{N, 1,1}$ и $\left(\mathbb{R}^{N, 1,1}\right)^{*}$ двойственны одно другому и получаются из $\mathbb{R}^{N+1,2 ~ “ з а б ы в а н и е м " ~} \mathbf{e}_{0}$ (соответственно $\left.\mathbf{e}_{\infty^{-}}\right)$компонент. Так, $\mathbb{R}^{N, 1,1}$ порождается $N+2$ линейно независимыми векторами $\mathbf{e}_{1}, \ldots, \mathbf{e}_{N}, \mathbf{e}_{N+3}, \mathbf{e}_{\infty}$ и снабжено вырожденной билинейной формой сигнатуры $(N, 1,1)$, относительно которой указанные выше векторы попарно ортогональны, первые $N$ из них пространственно-подобны: $\left\langle\mathbf{e}_{i}, \mathbf{e}_{i}\right\rangle=1$ для $1 \leqslant i \leqslant N$, в то время как последние два соответственно времениподобный и изотропный: $\left\langle\mathbf{e}_{N+3}, \mathbf{e}_{N+3}\right\rangle=-1$ и $\left\langle\mathbf{e}_{\infty}, \mathbf{e}_{\infty}\right\rangle=0$. Аналогично, $\left(\mathbb{R}^{N, 1,1}\right)^{*}$ снабжено ортогональным базисом из векторов $\mathbf{e}_{1}, \ldots, \mathbf{e}_{N}, \mathbf{e}_{N+3}, \mathbf{e}_{0}$, вновь с изотропным последним вектором: $\left\langle\mathbf{e}_{0}, \mathbf{e}_{0}\right\rangle=0$. Отметим, что один и тот же символ $\langle\cdot, \cdot\rangle$ используется для обозначения двух разных вырожденных билинейных форм на двух пространствах. Мы еще более расширим область применения этого символа, обозначая им (невырожденное) спаривание между этими двумя пространствами, которое устанавливается равенством $\left\langle\mathbf{e}_{0}, \mathbf{e}_{\infty}\right\rangle=-\frac{1}{2}$ в дополнение к предыдущим соотношениям. (Напомним, что вырожденная билинейная форма не может использоваться для отождествления векторного пространства со своим дуальным.)

В обеих вышеупомянутых моделях верно следующее:

- гиперплоскость $P=(v, d)$ моделируется точкой в пространстве $\mathbb{P}\left(\mathbb{R}^{N, 1,1}\right)$ с представителем

$$
\widehat{p}=v+2 d \mathbf{e}_{\infty}+\mathbf{e}_{N+3} ;
$$

- гиперсфера $S=($ c,$r)$ моделируется точкой в пространстве $\mathbb{P}\left(\left(\mathbb{R}^{N, 1,1}\right)^{*}\right)$ с представителем

$$
\widehat{s}=c+\mathbf{e}_{0}+r \mathbf{e}_{N+3} .
$$

Одна из этих двух моделей будет выделяться, если мы будем рассматривать одно из двух пространств как основное и интерпретировать точки второго как гиперплоскости в основном. В иилиндрической модели Бляшке основное пространство есть $\mathbb{P}\left(\mathbb{R}^{N, 1,1}\right)$, чьи точки моделируют гиперплоскости $P \subset \mathbb{R}^{N}$. Гиперсфера $S \subset \mathbb{R}^{N}$ тогда моделируется как гиперплоскость $\left\{\xi \in \mathbb{P}\left(\mathbb{R}^{N, 1,1}\right)\right.$ : $\langle\widehat{s}, \xi\rangle=0\}$ в пространстве $\mathbb{P}\left(\mathbb{R}^{N, 1,1}\right)$. Базовые свойства этой модели:

(i) Ориентированные гиперплоскости $P \subset \mathbb{R}^{N}$ взаимно однозначно соответствуют точкам $\widehat{p}$ квадрики $\mathbb{P}\left(\mathbb{L}^{N, 1,1}\right)$, где

$$
\mathbb{L}^{N, 1,1}=\left\{\xi \in \mathbb{R}^{N, 1,1}:\langle\xi, \xi\rangle=0\right\} .
$$


(ii) Две ориентированные гиперплоскости $P_{1}, P_{2} \subset \mathbb{R}^{N}$ ориентированно касаются (параллельны), если и только если их представители $\widehat{p}_{1}, \widehat{p}_{2}$ отличаются на вектор, параллельный $\mathbf{e}_{\infty}$.

(iii) Ориентированная гиперсфера $S \subset \mathbb{R}^{N}$ ориентированно касается ориентированной гиперплоскости $P \subset \mathbb{R}^{N}$, если и только если $\widehat{p} \in \widehat{s}$, т.е. $\langle\widehat{p}, \widehat{s}\rangle=0$. Тем самым гиперсфера $S$ интерпретируется как множество всех своих касательных гиперплоскостей.

Квадрика $\mathbb{P}\left(\mathbb{L}^{N, 1,1}\right)$ диффеоморфна иллиндру Бляшке

$$
\mathscr{Z}=\left\{(v, d) \in \mathbb{R}^{N+1}:|v|=1\right\}=\mathbb{S}^{N-1} \times \mathbb{R} \subset \mathbb{R}^{N+1} .
$$

Две точки этого цилиндра представляют параллельные гиперплоскости, если они лежат на одной прямолинейной образующей $\mathscr{Z}$, параллельной его оси. В объемлющем пространстве $\mathbb{R}^{N+1}$ цилиндра Бляшке ориентированные гиперсферы $S \subset \mathbb{R}^{N}$ находятся во взаимно однозначном соответствии с гиперплоскостями, не параллельными оси $\mathscr{Z}$ :

$$
S \sim\left\{(v, d) \in \mathbb{R}^{N+1}:\langle c, v\rangle-d-r=0\right\} .
$$

Пересечение такой гиперплоскости с $\mathscr{Z}$ состоит из точек на $\mathscr{Z}$, которые представляют касательные гиперплоскости к $S \subset \mathbb{R}^{N}$, как видим из уравнения (11).

В данной работе мы не будем использовать циклографическую модель геометрии Лагерра; ее краткое описание мы помещаем в приложении 1.

\section{4. Дискретные линии кривизны в геометриях Ли, Мёбиуса и Лагерра}

Начиная с данного раздела, мы ограничимся геометрией поверхностей в трехмерном евклидовом пространстве $\mathbb{R}^{3}$. Соответственно, необходимо положить $N=3$ во всех предыдущих рассмотрениях.

Естественно рассматривать следующие объекты как дискретные аналоги поверхностей в различных геометриях, обсуждавшихся выше.

- В геометрии Ли поверхность считается образованной своими контактными элементами. Эти контактные элементы интерпретируются как точки поверхности и ее касательные плоскости (или, что эквивалентно, нормали) в этих точках. Такое представление естественно дискретизуется: дискретная поверхность есть отображение

$$
(x, P): \mathbb{Z}^{2} \rightarrow\left\{\text { контактные элементы поверхностей в } \mathbb{R}^{3}\right\},
$$

или, в проективной модели геометрии Ли, отображение

$$
\ell: \mathbb{Z}^{2} \rightarrow \mathscr{L}_{0}^{4,2}
$$

где, напомним, $\mathscr{L}_{0}^{4,2}$ обозначает множество изотропных прямых в $\mathbb{P}\left(\mathbb{R}^{4,2}\right)$.

- В геометрии Мёбиуса поверхность считается просто составленной из своих точек. Дискретная поверхность есть отображение

$$
x: \mathbb{Z}^{2} \rightarrow \mathbb{R}^{3},
$$


или, в проективной модели, отображение

$$
\widehat{x}: \mathbb{Z}^{2} \rightarrow \mathbb{P}\left(\mathbb{L}^{4,1}\right) .
$$

- В геометрии Лагерра поверхность рассматривается как огибающая системы своих касательных плоскостей. Дискретная поверхность есть отображение

$$
P: \mathbb{Z}^{2} \rightarrow\left\{\text { ориентированные плоскости в } \mathbb{R}^{3}\right\},
$$

или, в проективной модели, отображение

$$
\widehat{p}: \mathbb{Z}^{2} \rightarrow \mathbb{P}\left(\mathbb{L}^{3,1,1}\right) .
$$

Следует упомянуть, что существенную роль в описании поверхности в геометрии Лагерра играет гауссово отображение

$$
v: \mathbb{Z}^{2} \rightarrow \mathbb{S}^{2}
$$

задаваемое единичными нормалями $v$ к касательным плоскостям $P=$ $(v, d)$.

Таким образом, описание поверхности в геометрии Ли содержит больше информации, чем описание поверхности в мёбиусовой или лагерровой геометрии. Фактически, оно охватывает оба последующих.

4.1. Геометрия Ли. Нижеследующее определение служит дискретизацией описания поверхностей, параметризованных линиями кривизны в геометрии Ли, которое можно найти, к примеру, в [24].

ОПРЕДЕлЕниЕ 18 (главные сети контактных элементов, евклидова модель). Отображение

$$
(x, P): \mathbb{Z}^{2} \rightarrow\left\{\text { контактные элементы поверхностей в } \mathbb{R}^{3}\right\}
$$

называется главной сетью контактных элементов, если любые два соседних контактных элемента $(x, P),\left(x_{i}, P_{i}\right)$ имеют общую сферу $S^{(i)}$, т.е. сферу, касающуюся обеих плоскостей $P, P_{i}$ в соответствующих точках $x, x_{i}$.

Таким образом, нормали к соседним плоскостям $P, P_{i}$ в соответствующих точках $x, x_{i}$ пересекаются в точке $c^{(i)}$ (центре сферы $S^{(i)}$ ), и расстояния от $c^{(i)}$ до $x$ и до $x_{i}$ равны, см. рис. 10 . Эти сферы $S^{(i)}$ ставятся в соответствие ребрам $\mathbb{Z}^{2}$, параллельным $i$-й координатной оси, и будут называться сферами главных кривизн дискретной поверхности.

Прямой перевод определения 18 на язык проективной модели дает:

ОПРЕДЕЛЕниЕ 19 (главная сеть контактных элементов, проективная модель). Отображение $\ell: \mathbb{Z}^{2} \rightarrow \mathscr{L}_{0}^{4,2}$ называется главной сетью контактных элементов, если оно является дискретной конгруэнцией изотропных прямых в $\mathbb{P}\left(\mathbb{R}^{4,2}\right)$, т.е. любые две соседние прямые пересекаются:

$$
\ell(u) \cap \ell\left(u+e_{i}\right)=\widehat{s}^{(i)}(u) \in \mathbb{P}\left(\mathbb{L}^{4,2}\right) \quad \forall u \in \mathbb{Z}^{2}, \quad \forall i=1,2 .
$$




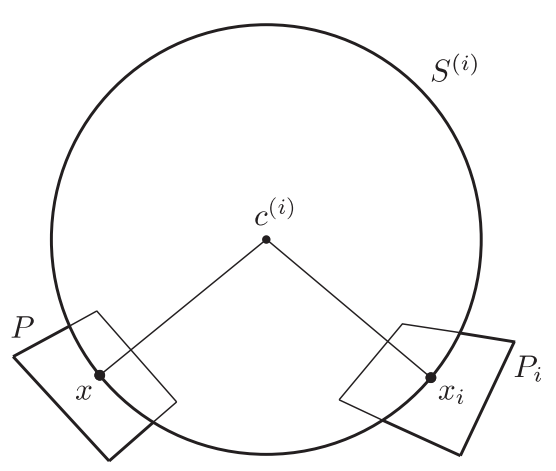

Рис. 10. Сферы главных кривизн

В проективной модели представители сфер главных кривизн $S^{(i)} i$-го координатного направления образуют соответствующую фокальную сеть конгруэнции прямых $\ell$;

$$
\widehat{s}^{(i)}: \mathbb{Z}^{2} \rightarrow \mathbb{P}\left(\mathbb{L}^{4,2}\right), \quad i=1,2,
$$

ср. с определением 11. В соответствии с теоремой 12 , обе фокальные сети являются $\mathrm{Q}$-сетями в $\mathbb{P}\left(\mathbb{R}^{4,2}\right)$. Это служит мотивировкой следующего определения.

ОПРЕДЕЛЕниЕ 20 (дискретная R-конгруэнция сфер). Отображение

$$
S: \mathbb{Z}^{m} \rightarrow\left\{\text { ориентированные сферы в } \mathbb{R}^{3}\right\}
$$

называется дискретной R-конгруэнцией сфер (конгруэнцией Рибокура), если соответствующее отображение

$$
\widehat{s}: \mathbb{Z}^{m} \rightarrow \mathbb{P}\left(\mathbb{L}^{4,2}\right)
$$

является Q-сетью в $\mathbb{P}\left(\mathbb{R}^{4,2}\right)$.

Геометрическое описание дискретных R-конгруэнций будет дано в разделе 5.

СлЕДСтвиЕ 21 (сферы кривизн образуют R-конгруэнцию). Для главной сети контактных элементов сферы главных кривизн $i$-го координатного направления $(i=1,2)$ образуют двумерную дискретную $R$-конгруэнцию.

Переходя к изучению преобразований главных сетей контактных элементов, введем следующее определение.

ОпРЕДЕлЕниЕ 22 (преобразования Рибокура, евклидова модель). Две главные сети контактных элементов

$$
(x, P),\left(x^{+}, P^{+}\right): \mathbb{Z}^{2} \rightarrow\left\{\text { контактные элементы поверхностей в } \mathbb{R}^{3}\right\}
$$

называются преобразованиями Рибокура друг друга, если любые два соответствующих контактных элемента $(x, P)$ и $\left(x^{+}, P^{+}\right)$имеют общую сферу $S$, т.е. сферу, которая касается обеих плоскостей $P, P^{+}$в соответствующих точKax $x, x^{+}$. 


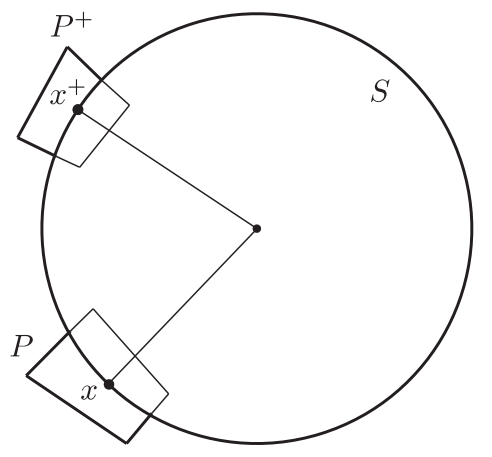

Рис. 11. Преобразование Рибокура

Вновь, непосредственно переводя определение 22 на язык проективной модели, получим:

ОПРЕДЕЛЕНИЕ 23 (преобразование Рибокура, проективная модель). Две главные сети контактных элементов $\ell, \ell^{+}: \mathbb{Z}^{2} \rightarrow \mathscr{L}_{0}^{4,2}$ называются преобразованиями Рибокура друг друга, если эти дискретные конгруэнции изотропных прямых находятся в отношении F-преобразования, т.е. если любая пара соответствующих прямых пересекается:

$$
\ell(u) \cap \ell^{+}(u)=\widehat{s}(u) \in \mathbb{P}\left(\mathbb{L}^{4,2}\right) \quad \forall u \in \mathbb{Z}^{2} .
$$

Сферы $S$ преобразования Рибокура разумно ассоциировать с вершинами $u$ решетки $\mathbb{Z}^{2}$ или, лучше, с “вертикальными” ребрами, соединяющими вершины $(u, 0)$ и $(u, 1)$ решетки $\mathbb{Z}^{2} \times\{0,1\}$. В проективной модели их представители

$$
\widehat{s}: \mathbb{Z}^{2} \rightarrow \mathbb{P}\left(\mathbb{L}^{4,2}\right)
$$

образуют фокальную сеть трехмерной конгруэнции прямых по третьему координатному направлению. Из теоремы 12 теперь получаем следующее утверждение.

СлЕДСтвиЕ 24 (сферы преобразования Рибокура образуют R-конгруэнцию). Сферы преобразования Рибокура общего положения образуют дискретную $R$-конгруэниию.

Обратимся теперь к изучению геометрии элементарного четырехугольника контактных элементов главной сети, состоящего из $\ell \sim(x, P), \ell_{1} \sim\left(x_{1}, P_{1}\right)$, $\ell_{2} \sim\left(x_{2}, P_{2}\right)$ и $\ell_{12} \sim\left(x_{12}, P_{12}\right)$.

Мы оставим в стороне вырожденный омбилический случай, когда все четыре прямые имеют общую точку и порождают четырехмерное пространство. Геометрически это означает, что заданы четыре контактных элемента одной сферы $S \subset \mathbb{R}^{3}$. В этой ситуации невозможно сделать никаких заключений относительно положения четырех точек $x, x_{1}, x_{2}, x_{12}$ на сфере $S$ : они совершенно произвольны. 
В неомбилической ситуации пространство, порожденное четырьмя прямыми $\ell, \ell_{1}, \ell_{2}, \ell_{12}$, трехмерное. Четыре элемента $\widehat{x}, \widehat{x}_{1}, \widehat{x}_{2}, \widehat{x}_{12} \in \mathbb{P}\left(\mathbb{L}^{4,2}\right)$, соответствующие точкам $x, x_{1}, x_{2}, x_{12} \in \mathbb{R}^{3}$, получаются как пересечения четырех изотропных прямых $\ell, \ell_{1}, \ell_{2}, \ell_{12}$ с проективной гиперплоскостью $\mathbb{P}\left(\mathbf{e}_{6}^{\perp}\right)$ в $\mathbb{P}\left(\mathbb{R}^{4,2}\right)$. Тем самым четыре элемента $\widehat{x}, \widehat{x}_{1}, \widehat{x}_{2}, \widehat{x}_{12}$ лежат в плоскости. Для изучения этой конфигурации хорошо подходит проективная модель мёбиусовой геометрии. Именно, опуская несущественную (нулевую) $\mathbf{e}_{6}$-компоненту, получаем плоский четырехугольник на мёбиусовой сфере $\mathbb{P}\left(\mathbb{L}^{4,1}\right)$. Пункт 4.2 посвящен изучению подобных объектов.

Аналогично, четыре элемента $\widehat{p}, \widehat{p}_{1}, \widehat{p}_{2}, \widehat{p}_{12} \in \mathbb{P}\left(\mathbb{L}^{4,2}\right)$, соответствующие плоскостям $P, P_{1}, P_{2}, P_{12} \in \mathbb{R}^{3}$, получаются как пересечения четырех изотропных прямых $\ell, \ell_{1}, \ell_{2}, \ell_{12}$ с проективной гиперплоскостью $\mathbb{P}\left(\mathbf{e}_{\infty}^{\perp}\right)$ в $\mathbb{P}\left(\mathbb{R}^{4,2}\right)$. Поэтому четыре элемента $\widehat{p}, \widehat{p}_{1}, \widehat{p}_{2}, \widehat{p}_{12}$ тоже лежат в плоскости. Для изучения подобной конфигурации в данном случае подходит геометрия Лагерра; это мы осуществим в п. 4.3 .

4.2. Геометрия Мёбиуса: циркулярные сети. Циркулярные сети были введены и изучены в контексте теории интегрируемых систем в [46], [14], [49].

Предостережение: в данном пункте $\widehat{x}$ обозначает представителя в геометрии Мёбиуса (т.е. в $\mathbb{L}^{4,1}$ ), а не представителя в $\mathbb{L}^{4,2}$ в геометрии Ли. Представитель в геометрии Мёбиуса получается из представителя в геометрии Ли вычеркиванием (нулевой) $\mathbf{e}_{6}$-компоненты.

Мы будем предполагать, что изучаемые нами главные сети контактных элементов - общего положения, т.е. не содержат омбилических четверок. Основной результат данного пункта состоит в следующем.

ТЕОРЕмА 25 (точки главной сети контактных элементов образуют циркулярную сеть). Для главной сети контактных элементов

$$
(x, P): \mathbb{Z}^{2} \rightarrow\left\{\text { контактные элементы поверхностей в } \mathbb{R}^{3}\right\}
$$

ее точки $x: \mathbb{Z}^{2} \rightarrow \mathbb{R}^{3}$ образуют циикулярную сеть.

В утверждении теоремы 25 встречается новое понятие, которое можно определить двумя разными способами.

ОПРЕДЕЛЕНиЕ 26 (циркулярная сеть, евклидова модель). Сеть $x: \mathbb{Z}^{m} \rightarrow \mathbb{R}^{3}$ называется циркулярной, если вершины каждого элементарного четырехугольника $\left(x, x_{i}, x_{i j}, x_{j}\right)$ (для любого элемента $u \in \mathbb{Z}^{m}$ и всех пар $1 \leqslant i \neq j \leqslant m$ ) лежат на окружности (в частности, компланарны).

ОПредЕЛЕНиЕ 27 (циркулярная сеть, проективная модель). Сеть $x: \mathbb{Z}^{m} \rightarrow$ $\mathbb{R}^{3}$ называется циркулярной, если соответствующие $\widehat{x}: \mathbb{Z}^{m} \rightarrow \mathbb{P}\left(\mathbb{L}^{4,1}\right)$ образуют Q-сеть в $\mathbb{P}\left(\mathbb{R}^{4,1}\right)$.

В данной ситуации перевод из евклидовой модели в проективную не очевиден и фактически составляет утверждение теоремы 25: в самом деле, эта теорема автоматически доказана (или, скорее, совершенно очевидна) в терминах определения 27, остается доказать эквивалентность определений 26 и 27. 
КОНЦЕПТУАЛЬНОЕ ДОКАЗАТЕЛЬСТвО. Линейное подпространство $\mathbb{R}^{4,1}$, порожденное изотропными векторами $\widehat{x}, \widehat{x}_{i}, \widehat{x}_{j}, \widehat{x}_{i j}$, трехмерно. Его ортогональное дополнение тем самым двумерно и лежит в $\mathbb{R}_{\text {out }}^{4,1}$. Поэтому оно представляет окружность (пересечение двух сфер).

ВЫЧИСЛИТЕЛЬНОЕ ДОКАЗАТЕЛЬСТВО. Для любых представителей $\widetilde{x} \in \mathbb{L}^{4,1}$ элементов $\widehat{x}$ условие, сформулированное в определении 27 , эквивалентно уравнению вида (1). Поскольку представители $\widehat{x}=x+\mathbf{e}_{0}+|x|^{2} \mathbf{e}_{\infty}$, заданные (14) лежат в аффинной гиперплоскости в $\mathbb{R}^{4,1}$ (их $\mathbf{e}_{0}$-компоненты равны 1 ), получаем для них уравнение вида (2). Ясно, что оно выполняется, если и только если $x$ задает Q-сеть в $\mathbb{R}^{3}$ и $|x|^{2}$ удовлетворяет тому же уравнению $(2)$, что и $x$. Покажем, что последнее условие эквивалентно циркулярности. На заданном плоском элементарном четырехугольнике $\left(x, x_{i}, x_{i j}, x_{j}\right)$ функция $|x|^{2}$ удовлетворяет уравнению (2) вместе с $|x-c|^{2}=|x|^{2}-2\langle x, c\rangle+|c|^{2}$ при любом $c \in \mathbb{R}^{3}$. Выберем в качестве $c$ центр окружности, проведенной через $x, x_{i}, x_{j}$, откуда $|x-c|^{2}=\left|x_{i}-c\right|^{2}=\left|x_{j}-c\right|^{2}$. Тогда уравнение (2) для $|x-c|^{2}$ превращается в $\left|x_{i j}-c\right|^{2}=|x-c|^{2}$, которое и означает, что $x_{i j}$ лежит на той же самой окружности. Теорема 25 доказана.

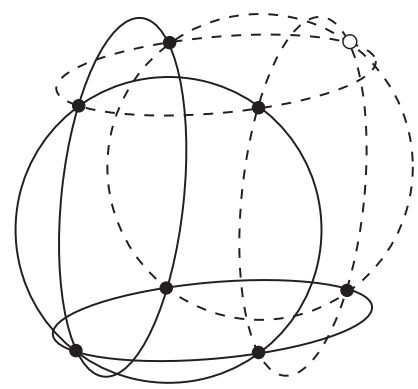

Рис. 12. Элементарный куб циркулярной сети

Двумерная циркулярная сеть $(m=2)$ есть дискретный аналог поверхности, параметризованной посредством линий кривизны, а для случая $m=3$ циркулярная сеть дает дискретизацию триортогональных систем координат в $\mathbb{R}^{3}$. Построение элементарного куба подобной циркулярной сети основано на следующей геометрической теореме.

ТЕОРЕма 28 (элементарный куб циркулярной сети). Пусть дано семь точек $x, x_{i}$ и $x_{i j} \quad(1 \leqslant i<j \leqslant 3)$ в $\mathbb{R}^{3}$ таких, что каждая из трех четверок $\left(x, x_{i}, x_{j}, x_{i j}\right)$ лежит на окружности $C_{i j}$. Определим три новых окружности $\tau_{i} C_{j k}$ тройками точек $\left(x_{i}, x_{i j}, x_{i k}\right)$ соответственно. Тогда эти новые окружности пересекаются в одной точке, см. рис. 12 :

$$
x_{123}=\tau_{1} C_{23} \cap \tau_{2} C_{31} \cap \tau_{3} C_{12} .
$$

ДокАЗАТЕЛьСтво. Это частный случай теоремы 15, примененной к квадрике $\mathbb{P}\left(\mathbb{L}^{4,1}\right)$. 
Теорема 28 может быть доказана и элементарно-геометрическими рассуждениями. Достаточно заметить, что в силу условий теоремы семь точек $x, x_{i}$, $x_{i j}$ лежат на двумерной сфере. Выполняя стереографическую проекцию этой сферы из полюса $x$, получаем плоскую конфигурацию, которая есть не что иное, как классическая теорема Микеля.

4.3. Геометрия Лагерра: конические сети. Конические сети были недавно введены в [2].

Предостережение: в данном пункте $\widehat{p}$ обозначает представителя в геометрии Лагерра (т.е. в $\mathbb{L}^{3,1,1}$ ), а не представителя в $\mathbb{L}^{4,2}$ в геометрии Ли. Представитель в геометрии Лагерра получается из представителя в геометрии Ли вычеркиванием (нулевой) $\mathbf{e}_{0}$-компоненты.

Как и в предыдущем пункте, мы предполагаем, что рассматриваемые главные сети контактных элементов не содержат омбилических четверок. Основной результат данного пункта состоит в следующем.

ТЕоремА 29 (касательные плоскости контактных элементов главной сети образуют коническую сеть). Для главной сети контактных элементов

$$
(x, P): \mathbb{Z}^{2} \rightarrow\left\{\text { контактные элементы поверхностей в } \mathbb{R}^{3}\right\}
$$

их касательные плоскости $P: \mathbb{Z}^{2} \rightarrow\left\{\right.$ ориентированные плоскости в $\left.\mathbb{R}^{3}\right\}$ образуют коническую сеть.

Вновь в утверждении теоремы встречается новое понятие, которое можно определить двумя разными способами.

ОПРЕДЕЛЕНиЕ 30 (коническая сеть, евклидова модель). Сеть

$$
P: \mathbb{Z}^{m} \rightarrow\left\{\text { ориентированные плоскости в } \mathbb{R}^{3}\right\}
$$

называется конической, если для любого $u \in \mathbb{Z}^{m}$ и всех пар $1 \leqslant i \neq j \leqslant m$ четыре плоскости $P, P_{i}, P_{i j}, P_{j}$ касаются конуса вращения (в частности, пересекаются в одной точке).

ОПРЕДЕЛЕНИЕ 31 (коническая сеть, проективная модель). Сеть

$$
P: \mathbb{Z}^{m} \rightarrow\left\{\text { ориентированные плоскости в } \mathbb{R}^{3}\right\}
$$

называется конической, если соответствующие $\widehat{p}: \mathbb{Z}^{m} \rightarrow \mathbb{P}\left(\mathbb{L}^{3,1,1}\right)$ образуют Q-сеть в $\mathbb{P}\left(\mathbb{R}^{3,1,1}\right)$.

Теорема 29 очевидна в терминах определения 31, т.е. ее реальное содержание состроит в переводе между языками евклидовой и проективной моделей, тем самым остается установить эквивалентность определений 30 и 31.

ДокАЗАтЕльство. Представители $\widehat{p}$ в (23) образуют Q-сеть, если и только если они удовлетворяют уравнению $(2)$, т.е. когда $v: \mathbb{Z}^{m} \rightarrow \mathbb{S}^{2}$ и $d: \mathbb{Z}^{m} \rightarrow \mathbb{R}$ удовлетворяют этому уравнению. Уравнение (2) на $v$ гарантирует, что $v: \mathbb{Z}^{m} \rightarrow$ $\mathbb{S}^{2}$ - действительно Q-сеть в $\mathbb{S}^{2}$, так что любой четырехугольник $\left(v, v_{i}, v_{i j}, v_{j}\right)$ в $\mathbb{S}^{2}$ плоский и тем самым его вершины лежат на окружности. Уравнение $(2)$ 
на $(v, d)$ гарантирует, что (единственная) точка пересечения плоскостей $P, P_{i}$, $P_{j}$ также лежит на $P_{i j}$ и все четыре плоскости пересекаются в одной точке. Таким образом, мы пришли к характеризации конических сетей в смысле определения 31 как таких сетей плоскостей, для которых любая четверка плоскостей $\left(P, P_{i}, P_{i j}, P_{j}\right)$ пересекается в одной точке и любой четырехугольник $\left(v, v_{i}, v_{i j}, v_{j}\right)$ единичных нормальных векторов плоский. Ясно, что это описание эквивалентно определению 30. Направление оси касательного конуса совпадает с центром окружности на сфере $\mathbb{S}^{2}$, описанной вокруг четырехугольника $\left(v, v_{i}, v_{i j}, v_{j}\right)$. Теорема 29 доказана.

Итак, конические сети суть Q-сети с циркулярным гауссовым отображением. Стоит упомянуть, что для задания конической сети достаточно задать циркулярное гауссово отображение $v: \mathbb{Z}^{m} \rightarrow \mathbb{S}^{2}$ и дополнительно числа $d$ (т.е. плоскости $P=(v, d))$ вдоль координатных осей $\mathbb{Z}^{m}$. Действительно, эти данные позволяют однозначно реконструировать коническую сеть. Это легко сделать индуктивной процедурой, элементарные шаги которой состоят в нахождении четвертой плоскости $P_{i j}$, если даны три плоскости $P, P_{i}, P_{j}$ и нормальный вектор $v_{i j}$ этой четвертой плоскости: $P_{i j}$ есть плоскость, перпендикулярная $v_{i j}$ и проходящая через точку пересечения плоскостей $P, P_{i}, P_{j}$.

4.4. Синтез. Ввиду теорем 25, 29, естественно выяснить, существует ли для данной циркулярной сети $x: \mathbb{Z}^{2} \rightarrow \mathbb{R}^{3}$ или конической сети

$$
P: \mathbb{Z}^{2} \rightarrow\left\{\text { ориентированные плоскости в } \mathbb{R}^{3}\right\}
$$

главная сеть контактных элементов

$$
(x, P): \mathbb{Z}^{2} \rightarrow\left\{\text { контактные элементы поверхностей в } \mathbb{R}^{3}\right\}
$$

с заданной половиной данных ( $x$ или $P$ ). Положительный ответ на этот вопрос - следствие приведенной ниже общей теоремы.

TЕорема 32 (расширение R-конгруэнций сфер до главных сетей контактных элементов). Пусть дана дискретная $R$-конгруэниия сळер

$$
S: \mathbb{Z}^{2} \rightarrow\left\{\text { ориентированные сферы в } \mathbb{R}^{3}\right\},
$$

тогда существует двухпараметрическое семейство главных сетей контактных элементов

$$
(x, P): \mathbb{Z}^{2} \rightarrow\left\{\text { контактные элементы поверхностей в } \mathbb{R}^{3}\right\}
$$

таких, что $S$ принадлежит контактному элементу $(x, P)$, т.е. $P$ - касательная плоскость $к S$ в точке $x \in S$, для всех $u \in \mathbb{Z}^{2}$. Такая главная сеть контактных элементов однозначно определяется заданием контактного элемента $(x, P)(0,0)$, содержащего сферу $S(0,0)$.

ДокАзАтельство. Пусть задана Q-сеть $\widehat{s}: \mathbb{Z}^{2} \rightarrow \mathbb{P}\left(\mathbb{L}^{4,2}\right)$ на квадрике Ли и мы ищем конгруэнцию изотропных прямых $\ell: \mathbb{Z}^{2} \rightarrow \mathscr{L}_{0}^{4,2}$ такую, что $\widehat{s}(u) \in$ $\ell(u)$ для всех $u \in \mathbb{Z}^{2}$. Построение начинается с выбора произвольной изотропной прямой $\ell(0,0)$, проходящей через $\widehat{s}(0,0)$, и основано на следующей лемме. 
Лемма 33. Для изотропной прямой $\ell \in \mathscr{L}_{0}^{4,2}$ и точки $\widehat{s}_{1} \in \mathbb{P}\left(\mathbb{L}^{4,2}\right)$, не лежащей на $\ell$, существует единственная изотропная прямая $\ell_{1}$, содержащая $\widehat{s}_{1}$ и пересекающая $\ell$.

ДоказАТЕЛЬство. Пусть $\widehat{s}, \widehat{\sigma}$ - две произвольные точки на $\ell$ (в однородных координатах), тогда прямая $\ell$ задается линейными комбинациями $\alpha \widehat{s}+\beta \widehat{\sigma}$. Соотношение $\left\langle\alpha \widehat{s}+\beta \widehat{\sigma}, \widehat{s}_{1}\right\rangle=0$ влечет

$$
\alpha: \beta=-\left\langle\widehat{\sigma}, \widehat{s}_{1}\right\rangle:\left\langle\widehat{s}_{,} \widehat{s}_{1}\right\rangle .
$$

Значит, существует единственная точка $\widehat{s}^{(1)} \in \ell$ такая, что $\left\langle\widehat{s}^{(1)}, \widehat{s}_{1}\right\rangle=0$. Теперь мы можем выбрать в качестве $\ell_{1}$ прямую, проходящую через $\widehat{s}_{1}$ и $\widehat{s}^{(1)}$. Лемма 33 доказана.

Вернемся к доказательству теоремы 32. С помощью леммы 33 мы можем построить изотропные прямые дискретной конгруэнции вдоль координатных осей решетки,

$$
\ell: \mathbb{Z} \times\{0\} \rightarrow \mathscr{L}_{0}^{4,2} \quad \text { и } \quad \ell:\{0\} \times \mathbb{Z} \rightarrow \mathscr{L}_{0}^{4,2}
$$

На следующем шаге необходимо продолжить конгруэнцию $\ell$ с координатных осей на все $\mathbb{Z}^{2}$. Элементарный шаг этого построения состоит в нахождении для трех заданных изотропных прямых $\ell, \ell_{1}, \ell_{2}$ (таких, что $\ell$ пересекает обе $\ell_{1}$ и $\ell_{2}$ ) четвертой, $\ell_{12}$, пересекающей $\ell_{1}$ и $\ell_{2}$ и проходящей через заданную точку $\widehat{s}_{12}$. Можно использовать для этого лемму 33 , но тогда необходимо показать, что это построение корректно, т.е. что прямая $\ell_{12}$, полученная из условия пересечения ее с $\ell_{1}$ и с $\ell_{2}$, одна и та же. Мы покажем это следующим образом. Пространство $V=\operatorname{span}\left(\ell, \ell_{1}, \ell_{2}\right)$ трехмерно. Точки $\widehat{s}, \widehat{s}_{1}, \widehat{s}_{2}$ лежат в $V$. По предположению теоремы, четырехугольник $\left(\widehat{s}, \widehat{s}_{1}, \widehat{s}_{12}, \widehat{s}_{2}\right)$ - плоский, следовательно, $\widehat{s}_{12}$ также лежит в $V$. Построим две плоскости в $V: \Pi_{1}=\operatorname{span}\left(\ell_{1}, \widehat{s}_{12}\right)$ и $\Pi_{2}=\operatorname{span}\left(\ell_{2}, \widehat{s}_{12}\right)$. Их пересечение есть прямая $\ell_{12}$, содержащая $\widehat{s}_{12}$. Остается доказать, что эта прямая - изотропная. Для этого заметим, что $\ell_{12}$ можно описать по-другому как прямую, определенную точками $\widehat{s}_{1}^{(2)}=\ell_{1} \cap \ell_{12}$ и $\widehat{s}_{2}^{(1)}=\ell_{2} \cap \ell_{12}$. Обе эти точки лежат в $\mathbb{P}\left(\mathbb{L}^{4,2}\right)$, поскольку они принадлежат изотропным прямым $\ell_{1}$ и $\ell_{2}$ соответственно. Но легко видеть, что прямая в $\mathbb{P}\left(\mathbb{R}^{4,2}\right)$, содержащая две точки $\mathbb{P}\left(\mathbb{L}^{4,2}\right)$, либо изотропная, либо не содержит других точек $\mathbb{P}\left(\mathbb{L}^{4,2}\right)$, в зависимости от того, полярны эти точки друг другу (относительно $\mathbb{P}\left(\mathbb{L}^{4,2}\right)$ ) или нет. В нашем случае прямая $\ell_{12}$ содержит по построению еще одну точку $\widehat{s}_{12}$ из $\mathbb{P}\left(\mathbb{L}^{4,2}\right)$, поэтому она должна быть изотропной. Теорема 32 доказана.

Поскольку представители $\widehat{x}$ в $\mathbb{P}\left(\mathbb{L}^{4,2}\right)$ циркулярной сети $x: \mathbb{Z}^{2} \rightarrow \mathbb{R}^{3}$ образуют $\mathrm{Q}$-сеть в $\mathbb{P}\left(\mathbb{R}^{4,2}\right)$ и то же самое верно для представителей $\widehat{p}$ в $\mathbb{P}\left(\mathbb{L}^{4,2}\right)$ конической сети $P: \mathbb{Z}^{2} \rightarrow\left\{\right.$ ориентированные плоскости в $\left.\mathbb{R}^{3}\right\}$, приходим к следующему заключению (независимо полученному Г. Поттманном [57]).

СлЕДСТВиЕ 34 (расширение циркулярных и конических сетей до главных сетей контактных элементов).

i) Пусть дана ииркулярная сеть $x: \mathbb{Z}^{2} \rightarrow \mathbb{R}^{3}$, тогда существует двухпараметрическое семейство конических сетей $P: \mathbb{Z}^{2} \rightarrow\left\{\right.$ плоскости в $\left.\mathbb{R}^{3}\right\}$ таких, 
что $x \in P$ для всех $u \in \mathbb{Z}^{2}$, с сеть контактных элементов

$$
(x, P): \mathbb{Z}^{2} \rightarrow\left\{\text { контактные элементы поверхнотей в } \mathbb{R}^{3}\right\}
$$

- главная. Подобная коническая сеть однозначно определяется заданием плоскости $P(0,0)$, проходящей через точку $x(0,0)$.

ii) Пусть дана коническая сеть $P: \mathbb{Z}^{2} \rightarrow\left\{\right.$ ориентированные плоскости в $\left.\mathbb{R}^{3}\right\}$, тогда существует двухпараметрическое семейство ииркулярных сетей $x: \mathbb{Z}^{2} \rightarrow \mathbb{R}^{3}$ таких, что $x \in P$ для всех $u \in \mathbb{Z}^{2}$, и сеть контактных элементов

$$
(x, P): \mathbb{Z}^{2} \rightarrow\left\{\text { контактные элементы поверхностей в } \mathbb{R}^{3}\right\}
$$

- главная. Подобная циркулярная сеть однозначно определяется заданием точки $x(0,0)$ в плоскости $P(0,0)$.

Эти соотношения отражены на рис. 13. Отметим, что оси конических сетей, соответствующих заданной циркулярной сети, совпадают с гауссовым отображением в их вершинах, рассмотренным В. К. Шифом [54].

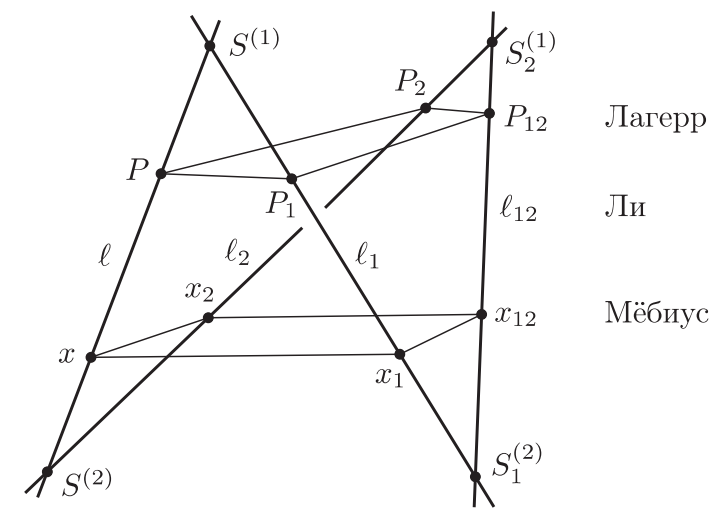

Рис. 13. Элементарный четырехугольник дискретной поверхности, параметризованной линиями кривизны с вершинами $x$ и касательными плоскостями $P$ в проективной модели. Вершины $x$ образуют циркулярную сеть (геометрия Мёбиуса) и лежат в плоскостях $P$, образующих коническую сеть (геометрия Лагерра). Контактные элементы $(x, P)$ представляются изотропными прямыми $\ell$ (геометрия Ли). Сферы главных кривизн $S^{(i)}$ проходят через пары соседних точек $x, x_{i}$ и касаются соответствующих пар плоскостей $P, P_{i}$

ЗАмЕЧАнИЕ. В ситуации, описанной в следствии 34, т.е. когда R-конгруэнция $S$ состоит из точек $x$ (и тем самым есть циркулярная сеть) или плоскостей $P$ (и тем самым есть коническая сеть), основной шаг построения леммы 33 допускает очень простое описание в евклидовой геометрии $\mathbb{R}^{3}$. Оно было дано Поттманном [57].

i) Пусть даны контактный элемент $(x, P)$ и точка $x_{1}$, требуется найти плоскость $P_{1}$, содержащую $x_{1}$ и такую, что существует сфера, $S^{(1)}$, 
касательная к обеим плоскостям $P, P_{1}$ в точках $x, x_{1}$ соответственно. Решение: $P_{1}$ получается из $P$ отражением относительно плоскости $\mathscr{P}$, ортогональной ребру $\left[x, x_{1}\right]$ и проходящей через его середину. Центр $c^{(1)}$ сферы $S^{(1)}$ находится как пересечение нормали к $P$ в $x$ с плоскостью $\mathscr{P}$.

ii) Пусть даны контактный элемент $(x, P)$ и плоскость $P_{1}$, требуется найти точку $x_{1}$ в $P_{1}$ такую, что существует сфера, $S^{(1)}$, касательная $\kappa$ обеим плоскостям $P, P_{1}$ в точках $x, x_{1}$ соответственно. Решение: точка $x_{1}$ получается из $x$ отражением относительно плоскости $\overline{\mathscr{P}}-$ биссектрисы двугранного угла, образованного $P, P_{1}$. Центр $c^{(1)}$ сферы $S^{(1)}$ находится как пересечение нормали к $P$ в $x$ и плоскости $\overline{\mathscr{P}}$.

\section{R-конгруэнции сфер}

В разделе 4 (следствия 21, 24) мы видели, что сферы главных кривизн главной сети контактных элементов, а также сферы преобразования Рибокура образуют дискретные R-конгруэнции, введенные в определении 20. В данном разделе мы будем изучать геометрию дискретных R-конгруэнций сфер. Определение 20 может быть переформулировано следующим образом: отображение

$$
S: \mathbb{Z}^{m} \rightarrow\left\{\text { ориентированные сферы в } \mathbb{R}^{3}\right\}
$$

или соответствующее отображение

$$
\widehat{s}: \mathbb{Z}^{m} \rightarrow \mathbb{L}^{4,2} \subset \mathbb{R}^{4,2}
$$

в пространстве однородных координат называется R-конгруэнцией сфер, если для любого $u \in \mathbb{Z}^{m}$ и любой пары $1 \leqslant i \neq j \leqslant m$ линейное подпространство

$$
\Sigma=\operatorname{span}\left(\widehat{s}, \widehat{s}_{i}, \widehat{s}_{j}, \widehat{s}_{i j}\right)
$$

трехмерно. Тем самым любому элементарному квадрату $\mathbb{Z}^{m}$ соответствует трехмерное линейное подпространство $\Sigma \subset \mathbb{R}^{4,2}$.

$\mathrm{R}$-конгруэнция сфер главных кривизн $S^{(i)}$ в $i$-м координатном направлении вырождена в том смысле, что подпространства элементарных четырехугольников

$$
\Sigma=\operatorname{span}\left(\widehat{s}^{(i)}, \widehat{s}_{i}^{(i)}, \widehat{s}_{i j}^{(i)}, \widehat{s}_{j}^{(i)}\right)
$$

содержат двумерные изотропные подпространства (отвечающие $\ell_{i}$ и $\ell_{i j}$ ). Rконгруэнции сфер для преобразований Рибокура общего положения, наоборот, невырождены: их подпространства $\Sigma$ не содержат двумерных изотропных подпространств и их элементарные четырехугольники включаются в планарные семейства сфер, которые мы вводим в следующем определении.

ОПРЕДЕЛЕНИЕ 35 (планарное семейство сфер). Планарное семейство сфер есть множество сфер, чьи представители $\widehat{s} \in \mathbb{P}\left(\mathbb{L}^{4,2}\right)$ содержатся в проективной плоскости $\mathbb{P}(\Sigma)$, где $\Sigma$ - трехмерное линейное подпространство $\mathbb{R}^{4,2}$ такое, что ограничение $\langle\cdot, \cdot\rangle$ на $\Sigma$ невырождено.

Таким образом, планарное семейство сфер есть пересечение $\mathbb{P}\left(\Sigma \cap \mathbb{L}^{4,2}\right)$. Ясно, что имеется две возможности: 
(a) $\left.\langle\cdot, \cdot\rangle\right|_{\Sigma}$ имеет сигнатуру $(2,1)$, так что сигнатура $\left.\langle\cdot, \cdot\rangle\right|_{\Sigma^{\perp}}$ также $(2,1)$;

(b) $\left.\langle\cdot, \cdot\rangle\right|_{\Sigma}$ имеет сигнатуру $(1,2)$, так что сигнатура $\left.\langle\cdot, \cdot\rangle\right|_{\Sigma^{\perp}}-(3,0)$.

Легко видеть, что планарное семейство однопараметрическое и параметризовано окружностью $\mathbb{S}^{1}$. Действительно, если $e_{1}, e_{2}, e_{3}$ задают ортогональный базис $\Sigma$ такой, что (например) $\left\langle e_{1}, e_{1}\right\rangle=\left\langle e_{2}, e_{2}\right\rangle=-\left\langle e_{3}, e_{3}\right\rangle=1$, то сферы планарного семейства образованы линейными комбинациями $\widehat{s}=\alpha_{1} e_{1}+\alpha_{2} e_{2}+e_{3}$ c

$$
\left\langle\alpha_{1} e_{1}+\alpha_{2} e_{2}+e_{3}, \alpha_{1} e_{1}+\alpha_{2} e_{2}+e_{3}\right\rangle=0 \quad \Leftrightarrow \quad \alpha_{1}^{2}+\alpha_{2}^{2}=1 .
$$

Во втором из упомянутых выше случаев пространство $\Sigma^{\perp}$ имеет тривиальное пересечение с $\mathbb{L}^{4,2}$, так что сферы планарного семейства $\mathbb{P}\left(\mathbb{L}^{4,2} \cap \Sigma\right)$ не имеют общих касательных сфер. Этот случай не имеет соответствия в гладкой дифференциальной геометрии. С точки зрения дискретной дифференциальной геометрии, первый случай более важен.

ОПРЕДЕЛЕНИЕ 36 (циклидное семейство сфер). Планарное семейство сфер называется циклидным, если $\left.\langle\cdot, \cdot\rangle\right|_{\Sigma}$ имеет сигнатуру $(2,1)$, так что сигнатура $\left.\langle\cdot, \cdot\rangle\right|_{\Sigma^{\perp}}$ также равна $(2,1)$.

Тем самым для циклидного семейства $\mathbb{P}\left(\mathbb{L}^{4,2} \cap \Sigma\right)$ существует дуалъное циклидное семейство $\mathbb{P}\left(\mathbb{L}^{4,2} \cap \Sigma^{\perp}\right)$ такое, что любая сфера первого семейства ориентированно касается любой сферы второго семейства. Однопараметрическое семейство сфер $\mathbb{P}\left(\mathbb{L}^{4,2} \cap \Sigma\right)$ имеет огибающую - каналовую поверхность в $\mathbb{R}^{3}$, и эта же поверхность служит огибающей дуального семейства $\mathbb{P}\left(\mathbb{L}^{4,2} \cap \Sigma^{\perp}\right)$. Подобные поверхности называются ииклидами Дюпена. Итак, каждому элементарному четырехугольнику дискретной R-конгруэнции, для которого сферы $\left(\widehat{s}, \widehat{s}_{i}, \widehat{s}_{i j}, \widehat{s}_{j}\right)$ порождают подпространство сигнатуры $(2,1)$, соответствует циклида Дюпена.

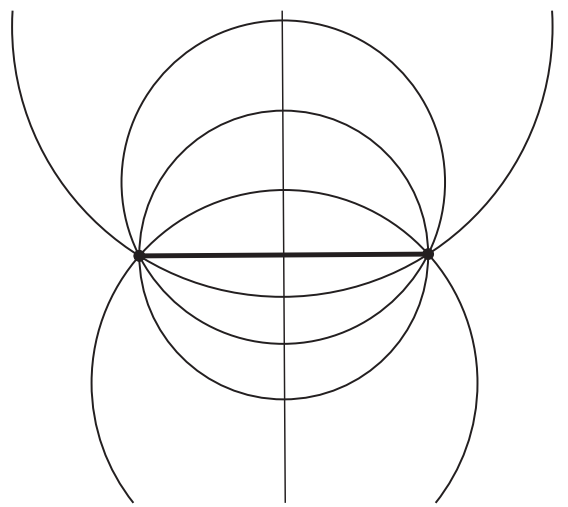

$a$

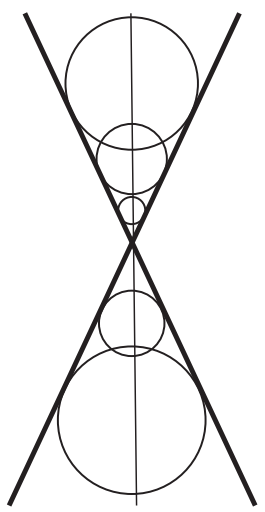

b

Рис. 14. а - циклидное семейство сфер, проходящих через окружность; $\mathrm{b}$ - циклидное семейство сфер, касающихся конуса

ПримеР. а) Точки окружности образуют планарное циклидное семейство сфер (нулевого радиуса). Дуальное семейство состоит из сфер, содержащих 
эту окружность, с центрами, лежащими на прямой, перпендикулярной плоскости окружности и проходящей через ее центр, см. рис. 14а. Соответствующая циклида Дюпена есть сама окружность. Можно доказать, что любая циклида Дюпена будет образом указанной в данном примере циклиды при некотором сферическом преобразовании Ли. Если мы рассмотрим циркулярную сеть как дискретную R-конгруэнцию, каждый ее элементарный четырехугольник несет такую же циклидную структуру.

b) Касательные плоскости к конусу вращения также образуют плоское циклидное семейство сфер. Дуальное семейство состоит из всех (ориентированных) сфер, касающихся конуса, см. рис. 14b. Соответствующая циклида Дюпена - сам конус. Если мы рассмотрим коническую сеть как дискретную Rконгруэнцию, каждый ее элементарный четырехугольник несет такую же циклидную структуру.

ТЕОРЕма 37 (общие касательные сферы двух соседних четырехугольников $\mathrm{R}$-конгруэнции). Для двух соседних четырехугольников дискретной $R$-конгруэниии сфер, несущих ииклидные семейства, в случае общего положения существует ровно две сферь, касающиеся всех шести сфер конгруэнции.

ДокАЗАТЕЛЬСтво. Пусть рассматриваемые нами четырехугольники принадлежат к планарным семействам, порожденным подпространствами $\Sigma_{1}$ и $\Sigma_{2}$ сигнатуры $(2,1)$. Эти четырехугольники имеют две общие сферы $\widehat{s}_{1}$ и $\widehat{s}_{2}$, которые порождают линейное пространство сигнатуры $(1,1)$. Каждое из планарных семейств $\Sigma_{1}$ и $\Sigma_{2}$ добавляет один пространственно-подобный вектор, поэтому линейное пространство $\Sigma_{1} \cup \Sigma_{2}$, порожденное всеми шестью сферами, четырехмерно и имеет сигнатуру $(3,1)$. Следовательно, его ортогональное дополнение $\left(\Sigma_{1} \cup \Sigma_{2}\right)^{\perp}$ двумерно и имеет сигнатуру $(1,1)$. Пересечение $\mathbb{L}^{4,2}$ с двумерным линейным подпространством сигнатуры $(1,1)$ содержит, после проективизации, ровно две сферы: в самом деле, если $e_{1}, e_{2}$ образуют ортогональный базис $\left(\Sigma_{1} \cup \Sigma_{2}\right)^{\perp} \mathrm{c}\left\langle e_{1}, e_{1}\right\rangle=-\left\langle e_{2}, e_{2}\right\rangle=1$, то сферы в этом пространстве соответствуют комбинациям $\alpha_{1} e_{1}+\alpha_{2} e_{2} \mathrm{c}$

$$
\left\langle\alpha_{1} e_{1}+\alpha_{2} e_{2}, \alpha_{1} e_{1}+\alpha_{2} e_{2}\right\rangle=0 \quad \Leftrightarrow \quad \alpha_{1}^{2}=\alpha_{2}^{2} \quad \Leftrightarrow \quad \alpha_{1}: \alpha_{2}= \pm 1 .
$$

Теорема 37 доказана.

В частности:

а) Для любых двух соседних четырехугольников циркулярной сети существует одна неориентированная сфера (тем самым две ориентированные сферы), содержащая обе окружности. Ее центр есть точка пересечения прямых, проходящих через центры окружностей перпендикулярно плоскостям, в которых они расположены, см. рис. 15a.

b) Для любых двух соседних четырехугольников конической сети существует единственная ориентированная сфера, касающаяся обоих конусов (вторая такая сфера - точка на бесконечности). Центр этой сферы есть точка пересечения осей конусов, см. рис. $15 \mathrm{~b}$.

Следующая теорема доказывается тем же способом, что и теорема 37. 

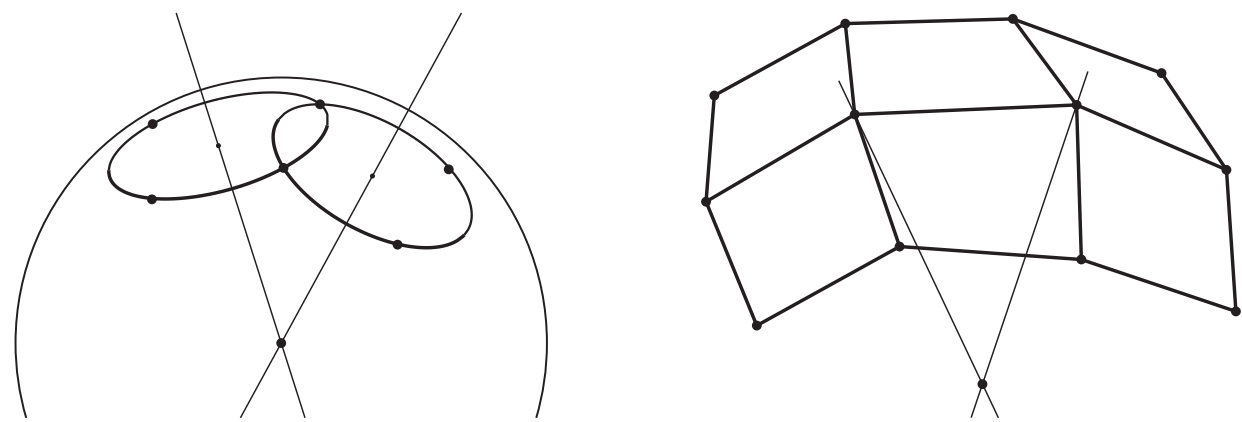

Рис. 15. а - нормали двух соседних четырехугольников циркулярной сети пересекаются: обе лежат в плоскости, ортогональной общему ребру и проходящей через его середину; b - оси конусов двух соседних четырехугольников конической сети пересекаются: две общие плоскости четырехугольников касаются обоих конусов, поэтому оси обоих конусов лежат в плоскости, делящей пополам двугранный угол, образованный этими двумя плоскостями

ТЕорема 38 (общие касательные сферы элементарного куба R-конгруэнции). Для элементарного трехмерного куба дискретной $R$-конгруэниии сфер, с ииклидными семействами сфер на каждой грани, в случае общего положения существуют в точности две сферъ, касающиеся всех восъми сфер, соответствующих вершинам куба.

Следует упомянуть, что эти сферы, соответствующие элементарным кубам, не образуют дискретной R-конгруэнции, вопреки утверждению, сформулированному А. Доливой в [62] в качестве основного результата.

Обратимся теперь к геометрической характеризации дискретных R-конгруэнций. Из уравнения (4) немедленно следует, что отображение

$$
S: \mathbb{Z}^{m} \rightarrow\left\{\text { ориентированные сферы в } \mathbb{R}^{3}\right\}
$$

является дискретной $\mathrm{R}$-конгруэнцией, если и только если центры сфер $c: \mathbb{Z}^{m} \rightarrow$ $\mathbb{R}^{3}$ образуют Q-сеть в $\mathbb{R}^{3}$, а две вещественные функции

$$
|c|^{2}-r^{2}: \mathbb{Z}^{m} \rightarrow \mathbb{R} \quad \text { и } \quad r: \mathbb{Z}^{m} \rightarrow \mathbb{R}
$$

удовлетворяют тому же уравнению вида (2), что и центры $c$. Опуская последнее уравнение на $r$, приходим к новому определению, менее ограничительному, чем определение R-конгруэнции. Фактически новое определение принадлежит мёбиусовой геометрии и использует обозначения и соглашения п. 3.2 (с $N=3$ ).

ОПРЕДЕЛЕНИЕ 39 (Q-конгруэнция сфер). Отображение

$$
S: \mathbb{Z}^{m} \rightarrow\left\{\text { неориентированные сферы в } \mathbb{R}^{3}\right\}
$$

называется Q-конгруэнцией сфер, если соответствующее отображение

$$
\widehat{s}: \mathbb{Z}^{m} \rightarrow \mathbb{P}\left(\mathbb{R}_{\text {out }}^{4,1}\right), \quad \widehat{s}=c+\mathbf{e}_{0}+\left(|c|^{2}-r^{2}\right) \mathbf{e}_{\infty},
$$


задает Q-сеть в $\mathbb{P}\left(\mathbb{R}^{4,1}\right)$.

Таким образом, отображение (36) дает Q-конгруэнцию, если и только если центры $c: \mathbb{Z}^{m} \rightarrow \mathbb{R}^{3}$ сфер $S$ образуют Q-сеть в $\mathbb{R}^{3}$, а функция $|c|^{2}-r^{2}$ удовлетворяет тому же уравнению (2), что и центры $c$.

Теорема 40 (характеризация R-конгруэнций среди Q-конгруэнций). Четыре (ориентированные) сберы $\left(S, S_{i}, S_{i j}, S_{j}\right)$ в $\mathbb{R}^{3}$ задают элементарный четырехугольник $R$-конгруэниии, если и только если они образуют (как неориентированные сферы) элементарный четырехуголъник $Q$-конгруэнции и удовлетворяют дополнительному условию:

(R) существует сфера ненулевого радиуса, ориентированно касающаяся всех четырех ориентированных сфер $S, S_{i}, S_{j}, S_{i j}$.

При выполнении этого условия любая сфера, ориентированно касающаяся трех сфер $S, S_{i}, S_{j}$, будет также ориентированно касаться четвертой $S_{i j}$.

ДокАЗАТЕЛЬство. Пусть $S_{0}$ - сфера с центром $c_{0}$ и (конечным) ориентированным радиусом $r_{0} \neq 0$, касающаяся $S, S_{i}, S_{j}$ с учетом ориентации. Это означает, что выполнены следующие условия:

$$
\left\langle c, c_{0}\right\rangle-\frac{1}{2}\left(|c|^{2}-r^{2}\right)-\frac{1}{2}\left(\left|c_{0}\right|^{2}-r_{0}^{2}\right)-r r_{0}=0
$$

(касание $S, S_{0}$, см. (10)) и два аналогичных уравнения, полученных заменой $(c, r)$ на $\left(c_{i}, r_{i}\right)$ и $\left(c_{j}, r_{j}\right)$. Теперь, используя тот факт, что $c$ и $|c|^{2}-r^{2}$ удовлетворяют одному и тому же уравнению вида (2), можем заключить, что уравнение $(38)$ выполнено для $\left(c_{i j}, r_{i j}\right)$, если и только если $r$ удовлетворяет тому же уравнению (2), что $c$ и $|c|^{2}-r^{2}$. Это доказывает теорему в случае, когда общая касательная сфера $S_{0}$ для трех сфер $S, S_{i}, S_{j}$ имеет конечный радиус. Случай, когда $S_{0}$ имеет бесконечный радиус, т.е. фактически есть плоскость, разбирается аналогично с помощью уравнения

$$
\left\langle c, v_{0}\right\rangle-r-d_{0}=0,
$$

которое будет играть роль уравнения (38). Теорема 40 доказана.

ЗАмЕчАниЕ. Как мы уже видели, в случае общего положения, если три ориентированные сферы $S, S_{i}, S_{j}$ имеют общую ориентированно касающуюся сферу, то они имеют однопараметрическое (циклидное) семейство общих соприкасающихся сфер, представленных трехмерным линейным подпространством $\Sigma$ в $\mathbb{R}^{4,2}$. Легко видеть, что если проекция $\Sigma$ на $\mathbf{e}_{\infty}^{\perp}$ не обращается в нуль, то семейство сфер, представленных $\Sigma^{\perp}$, содержит ровно две плоскости. (В случае конического семейства $\Sigma$ все элементы имеют нулевую $\mathbf{e}_{0}$-компоненту и представляют плоскости, тогда как $\Sigma^{\perp}$ не содержит плоскостей.) Поэтому во всех случаях, кроме конического, условие (R) можно заменить следующим:

$\left(\mathrm{R}_{0}\right)$ Четыре ориентированных сферы $S, S_{i}, S_{j}, S_{i j}$ имеют общую касательную плоскость (фактически, две общих касательных плоскости).

Обратимся к геометрической характеризации Q-конгруэнций. Она дается в следующей теореме. 
TЕОРемА 41 (три типа Q-конгруэнций). Четыре (неориентированные) сферы $\left(S, S_{i}, S_{i j}, S_{j}\right)$ в $\mathbb{R}^{3}$ образуют элементарный четырехугольник $Q$-конгруэниии, если и только если они удовлетворяют одному из следующих трех условиนั:

(i) они имеют общую перпендикулярную окружность, или

(ii) они пересекаются в паре точек, или

(iii) они пересекаются ровно по одной точке.

Случай (iii) можно рассматривать как вырождение как случая (i), так и (ii).

КонцЕПтУАЛЬНОЕ ДокАЗАТЕЛЬСТво. Предостережение: в данном доказательстве мы используем объекты геометрии Мёбиуса, отличные от объектов геометрии Ли с (буквально) теми же обозначениями. Линейное подпространство $\Sigma$ в $\mathbb{R}^{4,1}$, порожденное точками $\widehat{s}, \widehat{s}_{i}, \widehat{s}_{j}, \widehat{s}_{i j}$, трехмерно, и его ортогональное дополнение $\Sigma^{\perp}$ двумерно. Если $\Sigma^{\perp}$ лежит в $\mathbb{R}_{\text {out }}^{4,1}$, т.е. если ограничение метрики Минковского на $\Sigma^{\perp}$ положительно определено (имеет сигнатуру $(2,0))$, то $\Sigma^{\perp}$ представляет 1-сферу (окружность), перпендикулярную нашим четырем сферам, и мы имеем случай (i). Если же, наоборот, ограничение скалярного произведения на $\Sigma^{\perp}$ имеет сигнатуру $(1,1)$, так что $\Sigma$ лежит в $\mathbb{R}_{\text {out }}^{4,1}$, тогда $\Sigma$ представляет 0-сферу, которая является пересечением наших четырех сфер, и мы имеем случай (ii). Наконец, если ограничение скалярного произведения на $\Sigma^{\perp}$ вырождено, то $\Sigma \cap \Sigma^{\perp}$ есть изотропное одномерное линейное подпространство, которое представляет общую точку наших четырех сфер, и мы имеем случай (iii).

ВЫЧИСЛИТЕЛЬноЕ ДОКАЗАТЕЛЬСТВО. Четырехугольник в $\mathbb{R}^{3}$ с вершинами в центрах сфер $c, c_{i}, c_{j}, c_{i j}$ плоский; пусть П обозначает эту плоскость. Как и при доказательстве теоремы 25 , покажем, что существует точка $C \in \Pi$ такая, что

$$
|c-C|^{2}-r^{2}=\left|c_{i}-C\right|^{2}-r_{i}^{2}=\left|c_{j}-C\right|^{2}-r_{j}^{2}=\left|c_{i j}-C\right|^{2}-r_{i j}^{2} .
$$

Действительно, первые два из этих уравнений однозначно определяют $C$ как пересечение двух прямых $\ell_{i}$ и $\ell_{j}$ в П, где

$$
\ell_{i}=\left\{x \in \Pi:\left\langle 2 x-c_{i}-c, c_{i}-c\right\rangle=r^{2}-r_{i}^{2}\right\},
$$

тогда последнее уравнение в (40) автоматически выполнено. Если общее значение всех четырех выражений в (40) положительно (скажем, равно $R^{2}$ ), тогда эти четыре сферы ортогональны кругу в плоскости П с центром $C$ и радиусом $R$ и мы имеем случай (i), см. рис. 16. Если общее значение (40) отрицательно (скажем, равно $-R^{2}$ ), то пара точек на прямой, проходящей через $C$ перпендикулярно П, находящихся на расстоянии $R$ от $C$, принадлежит всем четырем сферам и мы имеем случай (ii). Наконец, если общее значение (40) равно 0, то $C$ есть точка пересечения всех четырех сфер и мы имеем случай (iii). Теорема 41 доказана.

Очевидно, что случай (i) Q-конгруэнций приводится к циклическим сетям, если радиусы всех сфер бесконечно малы, см. рис. 16. Q-конгруэнции с пересечениями типа (ii) будут естественными дискретными аналогами сферических конгруэнций, параметризованных вдоль главных направлений [37]. 


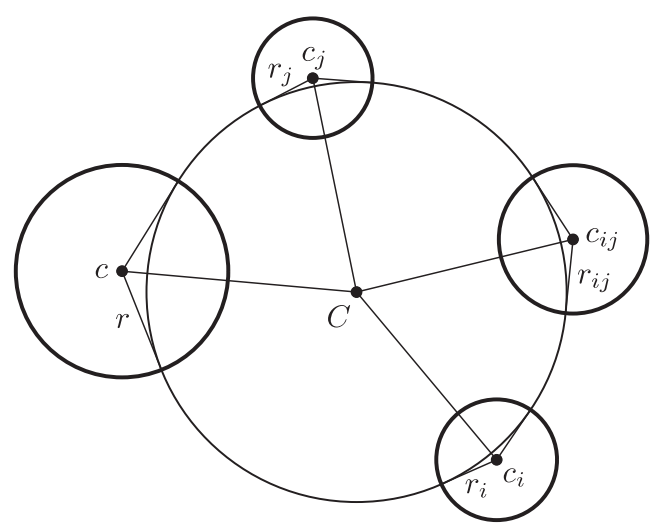

Рис. 16. Элементарный четырехугольник Q-конгруэнции сфер, случай ортогональной окружности

Сделаем еще несколько замечаний о Q-конгруэнциях сфер. Они многомерно-совместны при следующем ограничении: если заданы семь точек $\widehat{s}, \widehat{s}_{i}, \widehat{s}_{i j}$ в $\mathbb{P}\left(\mathbb{R}_{\text {out }}^{4,1}\right)$, то $\mathrm{Q}$-свойство (условие планарности) однозначно определяет восьмую точку $\widehat{s}_{123}$ в $\mathbb{P}\left(\mathbb{R}^{4,1}\right)$, которая, однако, может оказаться вне $\mathbb{P}\left(\mathbb{R}_{\text {out }}^{4,1}\right)$ и, тем самым, не будет определять вещественную сферу. Таким образом, соответствующая дискретная 3D-система корректно определена лишь на открытом подмножестве пространства начальных данных. В случае, если она определена, ее можно использовать для построения преобразований Q-конгруэнций с обычными свойствами перестановочности.

Отметим еще следующее различие Q-конгруэнций и R-конгруэнций: если даны три сферы $S, S_{i}, S_{j}$ элементарного четырехугольника, то имеется двухпараметрическое семейство четвертых сфер $S_{i j}$ в случае Q-конгруэнции и только однопараметрическое в случае R-конгруэнции. Это - следствие того факта, что $\mathbb{R}_{\text {out }}^{4,1}$ является открытым множеством в $\mathbb{R}^{4,1}$, в то время как $\mathbb{L}^{4,2}$ - гиперповерхность в $\mathbb{R}^{4,2}$.

\section{Приложение 1. Циклографическая модель геометрии Лагерра}

В циклографической модели геометрии Лагерра основное пространство пространство гиперсфер $\left(\mathbb{R}^{N, 1,1}\right)^{*}$, т.е. гиперсферы $S \subset \mathbb{R}^{N}$ моделируются точками $\widehat{s} \in \mathbb{P}\left(\left(\mathbb{R}^{N, 1,1}\right)^{*}\right)$, в то время как гиперплоскости $P \subset \mathbb{R}^{N}$ моделируются как гиперплоскости $\{\xi:\langle\widehat{p}, \xi\rangle=0\} \subset \mathbb{P}\left(\left(\mathbb{R}^{N, 1,1}\right)^{*}\right)$. Тем самым гиперплоскость $P$ интерпретируется как множество гиперсфер $S$, ориентированно касающихся $P$.

Приведем основные свойства этой модели.

(i) Множество ориентированных гиперсфер $S \subset \mathbb{R}^{N}$ взаимно однозначно отображается на множество точек

$$
\sigma=(c, r)
$$


пространства Минковского $\mathbb{R}^{N, 1}$, порожденного векторами $\mathbf{e}_{1}, \ldots, \mathbf{e}_{N}$, $\mathbf{e}_{N+3}$. Это пространство можно интерпретировать как аффинную часть $\mathbb{P}\left(\left(\mathbb{R}^{N, 1,1}\right)^{*}\right)$.

(ii) Ориентированные гиперплоскости $P \subset \mathbb{R}^{N}$ можно моделировать как гиперплоскости в $\mathbb{R}^{N, 1}$ :

$$
\pi=\left\{(c, r) \in \mathbb{R}^{N, 1}:\langle(v, 1),(c, r)\rangle=\langle v, c\rangle-r=d\right\} .
$$

Тем самым, ориентированные гиперплоскости $P \in \mathbb{R}^{N}$ будут взаимно однозначно соответствовать гиперплоскостям $\pi \subset \mathbb{R}^{N, 1}$, пересекающим подпространство $\mathbb{R}^{N}=\{(x, 0)\} \subset \mathbb{R}^{N, 1}$ под углом $\pi / 4$.

(iii) Ориентированная гиперсфера $S \subset \mathbb{R}^{N}$ ориентированно касается ориентированной гиперплоскости $P \subset \mathbb{R}^{N}$, если и только если $\sigma \in \pi$.

(iv) Две ориентированные гиперсферы $S_{1}, S_{2} \subset \mathbb{R}^{N}$ ориентированно касаются, если и только если их представители в пространстве Минковского $\sigma_{1}, \sigma_{2} \in \mathbb{R}^{N, 1}$ отличаются на изотропный вектор: $\left|\sigma_{1}-\sigma_{2}\right|=0$.

В циклографической модели группа преобразований Лагерра допускает следующее красивое описание.

ТЕорема 42 (фундаментальная теорема геометрии Лагерра). Групnа преобразований Лагерра изоморфна подгруппе афбиннъх преобразований $\mathbb{R}^{N, 1}$ вида $y \mapsto \lambda A y+b$, где $A \in O(N, 1), \lambda>0 u b \in \mathbb{R}^{N, 1}$.

\section{Список литературы}

[1] A. I. Bobenko, P. Schröder, "Discrete Willmore flow", Eurographics Symposium on Geometry Processing (Vienna, 2005), eds. M. Desbrun, H. Pottmann, 2005, 101-110.

[2] Y. Liu, H. Pottmann, J. Wallner, Y.-L. Yang, W. Wang, "Geometric modeling with conical meshes and developable surfaces", ACM Trans. Graphics, 25:3 (2006), 681689.

[3] А.Д. Александров, Выпуклые многогранники, Гостехиздат, М.-Л., 1950; англ. пер.: A. D. Alexandrov, Convex polyhedra, Springer Monogr. Math., Springer-Verlag, Berlin, 2005.

[4] А.В. Погорелов, Внешняя геометрия выпуклых поверхностей, Наука, М., 1969; англ. пер.: A. V. Pogorelov, Extrinsic geometry of convex surfaces, Transl. Math. Monogr., 35, Amer. Math. Soc., Providence, RI, 1973.

[5] R. Sauer, "Parallelogrammgitter als Modelle pseudosphärischer Flächen", Math. Z., 52 (1950), 611-622.

[6] W. Wunderlich, "Zur Differenzengeometrie der Flächen konstanter negativer Krümmung", Österreich. Akad. Wiss. Math.-Nat. Kl. S.-B. IIa, 160 (1951), 39-77.

[7] P. Alliez, D. Cohen-Steiner, O. Devillers, B. Lévy, M. Desbrun, "Anisotropic polygonal remeshing", ACM Trans. Graphics, 22:3 (2003), 485-493.

[8] S. Dong, S. Kircher, M. Garland, "Harmonic functions for quadrilateral remeshing of arbitrary manifolds", Comput. Aided Geom. Design, 22:5 (2005), 392-423.

[9] M. Marinov, L. Kobbelt, "Direct anisotropic quad-dominant remeshing", Computer graphics and applications, Proceedings of the 12th Pacific Conference, 2004, 207-216.

[10] R. Sauer, Differenzengeometrie, Springer-Verlag, Berlin, 1970.

[11] A. I. Bobenko, U. Pinkall, "Discrete surfaces with constant negative Gaussian curvature and the Hirota equation", J. Differential Geom., 43:3 (1996), 527-611. 
[12] A. I. Bobenko, U. Pinkall, "Discrete isothermic surfaces", J. Reine Angew. Math., 475 (1996), 187-208.

[13] A. Doliwa, P. M. Santini, "Multidimensional quadrilateral lattices are integrable", Phys. Lett. A, 233:4-6 (1997), 365-372.

[14] J. Cieśliński, A. Doliwa, P. M. Santini, "The integrable discrete analogues of orthogonal coordinate systems are multi-dimensional circular lattices", Phys. Lett. A, 235:5 (1997), 480-488.

[15] С. П. Новиков, И. А. Дынников, “Дискретные спектральные симметрии маломерных дифференциальных операторов и разностных операторов на правильных решетках и двумерных многообразиях", УМН, 52:5 (1997), 175-234; англ. пер.: S. P. Novikov, I. A. Dynnikov, "Discrete spectral symmetries of low-dimensional differential operators and difference operators on regular lattices and two-dimensional manifolds", Russian Math. Surveys, 52:5 (1997), 1057-1116.

[16] S.P. Novikov, "Schrödinger operators on graphs and symplectic geometry", The Arnoldfest (Toronto, ON, 1997), Fields Inst. Commun., 24, Amer. Math. Soc., Providence, RI, 1999, 397-413.

[17] С. П. Новиков, “Дискретный оператор Шрёдингера", Алгебра. Топология. Дифференииальные уравнения и их приложения, K 90-летию со дня рождения Л. С. Понтрягина, Тр. МИАН, 224, 1999, 275-290; англ. пер.: S.P. Novikov, "Difference Schrödinger operators", Proc. Steklov Inst. Math., 224 (1999), 250-265.

[18] I. A. Dynnikov, S. P. Novikov, "Geometry of the triangle equation on two-manifolds", Mosc. Math. J., 3:2 (2003), 419-438.

[19] A. I. Bobenko, U. Pinkall, "Discretization of surfaces and integrable systems", Discrete integrable geometry and physics (Vienna, 1996), Oxford Lecture Ser. Math. Appl., 16, eds. A. I. Bobenko, R. Seiler, Oxford Univ. Press, New York, 1999, 3-58.

[20] A. I. Bobenko, Yu. B. Suris, Discrete differential geometry. Consistency as integrability, arXiv: math.DG/0504358.

[21] A. I. Bobenko, Yu. B. Suris, "Integrable systems on quad-graphs", Int. Math. Res. Not., 11 (2002), 573-611.

[22] V.E. Adler, A.I. Bobenko, Yu.B. Suris, "Classification of integrable equations on quad-graphs. The consistency approach", Comm. Math. Phys., 233:3 (2003), 513543.

[23] A. Doliwa, "Quadratic reductions of quadrilateral lattices", J. Geom. Phys., 30:2 (1999), 169-186.

[24] W. Blaschke, Vorlesungen über Differentialgeometrie und geometrische Grundlagen von Einsteins Relativitätstheorie. III: Differentialgeometrie der Kreise und Kugeln, Springer-Verlag, Berlin, 1929.

[25] T. J. Willmore, Riemannian geometry, Oxford Sci. Publ., Oxford Univ. Press, New York, 1993.

[26] D. Ferus, K. Leschke, F. Pedit, U. Pinkall, "Quaternionic holomorphic geometry: Plücker formula, Dirac eigenvalue estimates and energy estimates of harmonic 2-tori", Invent. Math., 146:3 (2001), 507-593.

[27] И.А.Тайманов, “Двумерный оператор Дирака и теория поверхностей”, УМН, 61:1 (2006), 85-164; англ. пер.: I. А. Taimanov, "Two-dimensional Dirac operator and the theory of surfaces", Russian Math. Surveys, 61:1 (2006), 79-159.

[28] E. V. Ferapontov, "Lie sphere geometry and integrable systems", Tohoku Math. J. (2), $\mathbf{5 2 : 2}$ (2000), 199-233.

[29] E. V. Ferapontov, "Analog of Wilczynski's projective frame in Lie sphere geometry: Lie-applicable surfaces and commuting Schrödinger operators with magnetic fields", Internat. J. Math., 13:9 (2002), 959-985.

[30] E. Musso, L. Nicolodi, "On the Cauchy problem for the integrable system of Lie minimal surfaces", J. Math. Phys., 46:11 (2005), 113509, 15 p. 
[31] F. Burstall, U. Hertrich-Jeromin, "Harmonic maps in unfashionable geometries", Manuscripta Math., 108:2 (2002), 171-189.

[32] F.E. Burstall, U. Hertrich-Jeromin, "The Ribaucour transformation in Lie sphere geometry", Differential Geom. Appl., 24:5 (2006), 503-520.

[33] G. Darboux, Leçons sur la théorie générale des surfaces et les applications géométriques du calcul infinitésimal I-IV, Gauthier-Villars, Paris, 1914-1927.

[34] G. Darboux, Leçons sur les systèmes orthogonaux et les coordonnées curvilignes, Gauthier-Villars, Paris, 1910.

[35] L. Bianchi, Lezioni di geometria differenziale, vols. 1, 2, Enrico Spoerri, Pisa, 1922, 1923.

[36] Ch. Dupin, Développements de géométrie, Courcier, Paris, 1813.

[37] L.P. Eisenhart, Transformations of surfaces, Princeton Univ. Press, Princeton, NJ, 1923.

[38] L. Bianchi, "Le transformazioni di Ribaucour dei sistemi $n$-pli ortogonali e il teorema generale di permutabilità", Annali di Mat. (3), 27 (1918), 183-257.

[39] U. Hertrich-Jeromin, Introduction to Möbius differential geometry, London Math. Soc. Lecture Note Ser., 300, Cambridge Univ. Press, Cambridge, 2003.

[40] C. Rogers, W.K. Schief, Bäcklund and Darboux transformations. Geometry and modern applications in soliton theory, Cambridge Texts Appl. Math., Cambridge Univ. Press, Cambridge, 2002.

[41] Е.И. Ганжа, С.П. Царев, "Алгебраическая формула суперпозиции и полнота преобразований Бэклунда $(2+1)$-мерных интегрируемых систем", УМH, 51:6 (1996), 197-198; англ. пер.: E. I. Ganzha, S. P. Tsarev, "An algebraic formula for superposition and the completeness of the Backlund transformations of $(2+1)$ dimensional integrable systems", Russian Math. Surveys, 51:6 (1996), 1200-1202.

[42] V.E. Zakharov, "Description of the $n$-orthogonal curvilinear coordinate systems and Hamiltonian integrable systems of hydrodynamic type. I: Integration of the Lamé equations", Duke Math. J., 94:1 (1998), 103-139.

[43] И. М. Кричевер, “Алгебро-геометрические $n$-ортогональные криволинейные системы координат и решения уравнений ассоциативности", Функи. анализ и его прилож., 31:1 (1997), 32-50; англ. пер.: I. M. Krichever, "Algebraic-geometric $n$ orthogonal curvilinear coordinate systems and solutions of the associativity equations", Funct. Anal. Appl., 31:1 (1997), 25-39.

[44] B. Dubrovin, "Integrable systems in topological field theory", Nuclear Phys. B, 379:3 (1992), 627-689.

[45] A. W. Nutbourne, R. R. Martin, Differential geometry applied to curve and surface design. Vol. 1. Foundations, Ellis Horwood Ltd., Chichester; Halsted Press, New York, 1988.

[46] A. I. Bobenko, "Discrete conformal maps and surfaces", Symmetries and integrability of difference equations (Canterbury, 1996), London Math. Soc. Lecture Note Ser., 255, Cambridge Univ. Press, Cambridge, 1999, 97-108.

[47] М.Берже, Геометрия, в 2-х т., Мир, М., 1984; пер. с фр.: М. Berger, Géométrie, V. I-V, CEDIC, Paris; Nathan Information, Paris, 1977.

[48] A. I. Bobenko, D. Matthes, Yu. B. Suris, "Discrete and smooth orthogonal systems: $C^{\infty}$-approximation", Int. Math. Res. Not., 2003:45 (2003), 2415-2459.

[49] B. G. Konopelchenko, W. K. Schief, "Three-dimensional integrable lattices in Euclidean spaces: conjugacy and orthogonality", Proc. R. Soc. Lond. Ser. A Math. Phys. Eng. Sci., 454:1980 (1998), 3075-3104.

[50] A. Doliwa, S. V. Manakov, P. M. Santini, " $\bar{\partial}$-reductions of the multidimensional quadrilateral lattice. The multidimensional circular lattice", Comm. Math. Phys., 196:1 (1998), 1-18. 
[51] А. А. Ахметшин, Ю. С. Вольвовский, И. М. Кричевер, “Дискретные аналоги метрик Дарбу-Егорова", Солитоны, геометрия, топология - на перекрестках, $\mathrm{K}$ 60-летию со дня рождения С. П. Новикова, Тр. МИАН, 255, 1999, 21-45; англ. пер.: A. A. Akhmetshin, Y.S. Volvovski, I. M. Krichever, "Discrete analogues of the Darboux-Egorov metrics", Proc. Steklov Inst. Math., 255:2 (1999), 16-39.

[52] A. I. Bobenko, U. J. Hertrich-Jeromin, "Orthogonal nets and Clifford algebras", Proceedings of the Fifth Pacific Rim Geometry Conference (Sendai, 2000), Tohoku Math. Publ., 20, Tohoku Univ., Sendai, 2001, 7-22.

[53] W. K. Schief, "On the unification of classical and novel integrable surfaces. II: Difference geometry", Proc. R. Soc. Lond. Ser. A Math. Phys. Eng. Sci., 459:2030 (2003), 373-391.

[54] W.K. Schief, "On a maximum principle for minimal surfaces and their integrable discrete counterparts", J. Geom. Phys., 56:9 (2006), 1484-1495.

[55] A. Doliwa, P. M. Santini, M. Mañas, "Transformations of quadrilateral lattices", J. Math. Phys., 41:2 (2000), 944-990.

[56] A. Doliwa, "Discrete asymptotic nets and $W$-congruences in Plücker line geometry", J. Geom. Phys., 39:1 (2001), 9-29.

[57] H. Pottmann, Generalized principal meshes and their support structures, Geometry preprint 158, Technische Universität Wien, 2006.

[58] W. Blaschke, Projektive Geometrie, 3rd ed., Birkhäuser, Basel-Stuttgart, 1954.

[59] T. E. Cecil, Lie sphere geometry, Springer-Verlag, New York, 1992.

[60] W. Benz, Geometrische Transformationen, Bibliographisches Institut, Mannheim, 1992.

[61] H. Pottmann, M. Peternell, "Applications of the Laguerre geometry in CAGD", Comput. Aided Geom. Design, 15:2 (1998), 165-186.

[62] A. Doliwa, "The Ribaucour congruences of spheres within Lie sphere geometry", Bäcklund and Darboux transformations. The geometry of solutions (Halifax, NS, 1999), CRM Proc. Lecture Notes, 29, Amer. Math. Soc., Providence, RI, 2001, 159-166.

А. И. Бобенко

Institut für Mathematik, Technische Universität Berlin

E-mail: bobenko@math.tu-berlin.de

Ю. Б. Сурис

Zentrum Mathematik, Technische Universität München

E-mail: suris@ma.tum.de
Поступила в редакцию

20.11.2006 